\title{
Search for Magnetic Monopoles and Stable High-Electric-Charge Objects in 13 Tev Proton-Proton Collisions with the ATLAS Detector
}

\author{
G. Aad et al. \\ (ATLAS Collaboration)
}

(Received 27 May 2019; revised manuscript received 26 November 2019; published 23 January 2020)

\begin{abstract}
A search for magnetic monopoles and high-electric-charge objects is presented using $34.4 \mathrm{fb}^{-1}$ of $13 \mathrm{TeV} p p$ collision data collected by the ATLAS detector at the LHC during 2015 and 2016. The considered signature is based upon high ionization in the transition radiation tracker of the inner detector associated with a pencil-shape energy deposit in the electromagnetic calorimeter. The data were collected by a dedicated trigger based on the tracker high-threshold hit capability. The results are interpreted in models of Drell-Yan pair production of stable particles with two spin hypotheses $(0$ and $1 / 2)$ and masses ranging from 200 to $4000 \mathrm{GeV}$. The search improves by approximately a factor of 5 the constraints on the direct production of magnetic monopoles carrying one or two Dirac magnetic charges and stable objects with electric charge in the range $20 \leq|z| \leq 60$ and extends the charge range to $60<|z| \leq 100$.
\end{abstract}

DOI: 10.1103/PhysRevLett.124.031802

The symmetry between electric and magnetic charge in Maxwell's equations and the explanation for electric charge quantization resulting from Dirac's quantum description of the magnetic monopole $[1,2]$ are compelling arguments for its existence. Neither the spin nor the mass of a Dirac monopole is theoretically constrained. While monopoles appearing in grand unification theories $[3,4]$ typically have masses of the order of the unification scale $\left(m \sim 10^{16} \mathrm{GeV}\right)$, some extensions of the standard model predict electroweak monopoles with masses as low as $4 \mathrm{TeV}$ [5-9]. TeV-mass monopoles can be produced in the early Universe thermally or via the Kibble mechanism [10,11] in cosmological scenarios with a low reheat temperature after inflation [12].

Dirac's argument predicts the fundamental magnetic charge to be $q_{m}=N g_{D} e c$ (In this definition, $q_{m}$ is in SI units and $g_{D}$ is a dimensionless quantity.), where $g_{D}=$ $1 /(2 \alpha)=68.5$ is the Dirac charge, $\alpha$ is the fine structure constant, $N$ is an integer number, $e$ is the unsigned electron charge, and $c$ is the speed of light in vacuum. This implies that a high-velocity Dirac monopole of magnetic charge $|g|=g_{D}$ would interact with matter in a manner similar to that of an ion of electric charge $|z|=68.5$, where $z$ is in units of $e$. Since the energy loss is proportional to the square of the charge, a monopole with $|g|=g_{D}$ would deposit 4700 times more energy by ionization than a proton. The high stopping power also results in the

\footnotetext{
*Full author list given at the end of the article.

Published by the American Physical Society under the terms of the Creative Commons Attribution 4.0 International license. Further distribution of this work must maintain attribution to the author(s) and the published article's title, journal citation, and DOI. Funded by SCOAP .
}

production of a large number of $\delta$ rays. These two features result in a high-ionization signature that is also expected in the case of exotic stable high-electric-charge objects (HECOs), which may include, for example, aggregates of $u d$ - [13] or $s$-quark matter [14], $Q$ balls [15,16], and micro black-hole remnants [17].

This Letter presents a search for magnetic monopoles and HECOs, collectively referred to as highly ionizing particles, or HIPs, using $34.4 \mathrm{fb}^{-1}$ of $13 \mathrm{TeV}$ proton-proton $(p p)$ collision data collected by the ATLAS detector at the CERN Large Hadron Collider (LHC) during 2015 and 2016. Events containing at least one high-ionization object are selected. The results are interpreted in models of spin-0 and spin-1/2 Drell-Yan pair production of stable particles carrying one or two Dirac magnetic charges or an electric charge in the range $20 \leq|z| \leq 100$ with masses ranging from 200 to $4000 \mathrm{GeV}$.

Should monopoles exist in the mass range accessible to a particle accelerator, they could be copiously produced at the LHC. If they were detected, the measured mass and coupling would severely restrict cosmological scenarios. Since the numerous searches for monopoles of cosmological origin in cosmic rays and in matter $[18,19]$ have limited sensitivity to TeV-mass HIPs, the cross-section limits for low-mass HIPs from searches at colliders [20-34] are 6-9 orders of magnitude more stringent. The first LHC searches for HECOs and monopoles were made by the ATLAS Collaboration in $8 \mathrm{TeV} p p$ collisions $[24,25]$ by exploiting the high-ionization signature. The higher collision energy, the 5 times larger dataset and improvements in the trigger extend the sensitivity of the present search. While the previous search studied $|g| \leq 1.5 g_{D}$, the present search considers monopoles up to $|g|=2 g_{D}$, which are motivated by Schwinger, who showed that $N$ must be even for particles 
possessing both electric and magnetic charge [35-37]. The ATLAS monopole searches [25,27] are complementary to those performed using the dedicated MoEDAL experiment [28-31], which uses an induction technique to detect the magnetic flux of monopoles trapped in matter. While MoEDAL is sensitive to magnetic charges up to $5 g_{D}$, the present ATLAS search is able to set significantly better cross-section constraints for $1 g_{D}$ and $2 g_{D}$, the charge range in which it has a good acceptance [38].

Unlike searches using the induction technique, the present search is sensitive to high-charge HECOs in addition to monopoles. It is complementary to the low-charge HECO searches performed by ATLAS $(2 \leq|z| \leq 7[26,32,34])$ and CMS $(2 \leq|z| \leq 8$ [33]), all of which used muon triggers. A muon trigger is not appropriate for high-charge HECOs, which typically stop in the electromagnetic calorimeter, due to the charge-square dependence of $d E / d x$. In LHC run 1 , ATLAS probed electric charges up to $|z|=60[24,27]$ via the high-ionization signature. The present analysis is able to probe HECOs up to $|z|=100$, thereby reaching the previously unexplored charge range predicted for $u d$-quark matter [13].

The present search exploits the very characteristic highionization signature of HIPs in the ATLAS detector [39]. The ATLAS transition radiation tracker (TRT), which is the outermost tracker of the inner detector, consists of a barrel $(|\eta|<1.0$ and radius $0.563 \mathrm{~m}<r<1.066 \mathrm{~m})$ [ATLAS uses a right-handed coordinate system with its origin at the nominal interaction point (IP) in the center of the detector and the $z$ axis coinciding with the axis of the beam pipe. The $x$ axis points from the IP to the center of the LHC ring, and the $y$ axis points upward. Cylindrical coordinates $(r, \phi)$ are used in the transverse plane, $\phi$ being the azimuthal angle around the beam pipe. The pseudorapidity is defined in terms of the polar angle $\theta$ as $\eta=-\ln \tan (\theta / 2)$.] with 4-mm-diameter straws oriented parallel to the beam line, and two end caps $(0.77<|\eta|<2.0)$ with straws oriented radially. In LHC run 2, 56\% of the straws were filled with xenon gas while the others were filled with argon gas. Energy deposits in a TRT straw greater than $200 \mathrm{eV}$, called low-threshold (LT) hits, are used for tracking. The highthreshold (HT) hits, which result from energy deposits exceeding $6 \mathrm{keV}$ in $\mathrm{Xe}(2 \mathrm{keV}$ in $\mathrm{Ar})$ are typically used for electron identification, but can also indicate the presence of a highly ionizing particle. A 2 T superconducting solenoid surrounds the TRT. The lead/liquid-Ar (LAr) barrel electromagnetic (EM) calorimeter lies outside the solenoid in the $|\eta|<1.475$ region. It is divided into three shower-depth layers: EM1, EM2, and EM3, with accordion-shape electrodes and lead absorbers. The EM2 layer has the largest sampling depth (16 to $20 X_{0}$ ); its cell granularity is $\Delta \eta \times \Delta \phi=0.025 \times 0.025$. In the $|\eta|<1.8$ region, an additional presampler layer is used to measure the energy lost in front of the calorimeter. As a HIP traverses the TRT, a localized region of high ionization density with many HT hits from both the HIP and the $\delta$ rays is produced in its wake. HIPs slow down (and usually stop) in the EM calorimeter, where they leave a pencil-shape energy deposit since they do not induce a shower, being much heavier than the electron.

Signal efficiency estimates rely heavily on simulations, which use the GEANT4 framework [40,41] to model the HIP propagation and behavior in ATLAS. The detector simulation includes the full ATLAS geometry, descriptions of monopole acceleration in the solenoidal magnetic field, ionization energy losses in matter [42-45], $\delta$-electron production along the HIP trajectory, and a model accounting for electron-ion recombination in the LAr EM calorimeter [46]. For monopoles, the trajectory in the solenoidal magnetic field is straight in the $r-\phi$ plane and bends in the $r-z$ plane and the Bethe-Bloch formula is modified $[42,43]$ to account for the velocity-dependent Lorentz force. The interaction of HIPs with matter is independent of HIP spin.

The Drell-Yan (DY) pair-production process is used to estimate the kinematic distributions and cross sections of spin- 0 and spin- $1 / 2$ HIPs in the relevant ranges of charge and mass. The MadGraph5_aMC@NLO [47] event generator was used to model leading-order HIP DY pair production from the initial $p p$ state via quark-antiquark annihilation into a virtual photon. The charge-squared dependence of the HIP coupling to the photon implies divergences in the perturbative expansion beyond leading order [48]. PYTHIA version $8.212[49,50]$ was employed for the hadronization and the underlying-event generation, using the NNPDF2.3 [51] parton distribution functions of the proton with the A14 [52] set of tuned parameters ("tune"). The A2 [53] tune was used for the "pileup," which are additional simulated $p p$ collisions overlaid on each event according to the distribution of the number of $p p$ interactions per bunch crossing, $\mu$, in the data. Fully simulated HIP Monte Carlo (MC) samples are computationally intensive due to the high ionization. To minimize the number of such MC samples, model-independent efficiency maps, finely binned in kinetic energy and $|\eta|$, are produced from fully simulated single-particle samples of a given mass and charge. The spin-0 DY HIP four vectors from the generator are used to sample the maps in order to derive the spin-0 DY HIP selection efficiencies. The DY spin- $1 / 2$ selection efficiencies are derived from fully simulated DY samples, which are also used to validate the results obtained by sampling the efficiency maps and to assign a modeling uncertainty. The selection efficiencies for spin-0 DY HIPs are higher than for spin- $1 / 2$ because they have more central $\eta$ and harder kinetic energy distributions.

Incomplete knowledge of the simulation parameters translates into systematic uncertainties in the signal efficiencies. These uncertainties are estimated by varying the parameters within a range corresponding to an uncertainty of 1 standard deviation, as described in more detail in 
Ref. [27]. The dominant uncertainties are those due to the descriptions of the detector material and of electron-ion recombination in the EM calorimeter, each with an average relative uncertainty of $7 \%$. Other relevant parameters include the dependence of the multiplicity of TRT LT hits on pileup $\mu$, the HIP energy-loss calculation as well as the yield and range of $\delta$ electrons, and the fraction of energy cross talk between adjacent EM calorimeter cells. Additional uncertainties unrelated to detector effects include the uncertainty from MC statistics and the systematic uncertainty due to imprecise modeling or deriving DY efficiencies from the single-particle maps.

ATLAS uses a two-level trigger system [54]. Level 1 is a hardware-based trigger that defines calorimeter "regions of interest" (ROI). A dedicated software-based high-level trigger (HLT), which imposes requirements on the number and fraction of TRT HT hits in a narrow region around the ROI, started collecting data in October 2015. The level-1 seed requires transverse energy $E_{T}>22 \mathrm{GeV}$ in the electromagnetic calorimeter. ROI candidates with $E_{T}<50 \mathrm{GeV}$ in conjunction with an $E_{T}$-dependent minimum of 1 to $2 \mathrm{GeV}$ of energy in the hadronic calorimeter are vetoed. This unavoidable hadronic veto requirement, which improves rejection of hadrons in analyses using electrons and photons, limits the sensitivity to low-charge HIPs, which often penetrate to the hadronic calorimeter. In contrast to the $8 \mathrm{TeV}$ search [27], this veto on an energy deposit in the hadronic calorimeter is not applied to ROI candidates with $E_{T}>50 \mathrm{GeV}$, thereby increasing the acceptance for the HIPs that do not stop in the EM calorimeter. Following the level-1 trigger, the HLT is used to select HIP candidates with HT hits in the TRT. The counting is done in an $r-\phi$ wedge of size $10 \mathrm{mrad}$, where two trigger variables, $N_{\mathrm{HT} \text {,trig }}$ and $f_{\mathrm{HT}, \text { trig }}$, are used to define the number and fraction of TRT HT hits, respectively. The HLT selection criteria are defined as $N_{\mathrm{HT} \text {,trig }}>30$ and $f_{\mathrm{HT} \text {,trig }}>0.5$ to control the rate while maintaining high signal efficiency for high-charge HIPs. In addition, a pseudorapidity requirement of $|\eta|<1.7$ is applied to avoid the forward regions, which contain more backgrounds due to multijet events producing a higher rate of level-1 trigger seeds.

In this search, the HIP signal sensitivity is governed by the DY kinematic distributions and the dependence of the stopping power on two variables: the square of the charge and the velocity relative to the speed-of-light $\beta$. For HECOs, $d E / d x$ is proportional to $1 / \beta^{2}$ whereas it varies as $\ln \beta^{2}$ for monopoles $[42,43]$. The main source of signal loss is due to HIPs failing to produce a level-1 trigger ROI, because they either stop before the EM calorimeter (e.g., high-charge HIPs), deposit too little energy in the calorimeter or penetrate to the hadronic calorimeter and invoke the veto (e.g., low-charge HIPs). The probability for a HIP from DY pair production to induce a level-1 trigger signal is around $60 \%$ for HECOs with $|z|=20$ and $55 \%$ for monopoles with $|g|=g_{D}$ and decreases to $18 \%$ for $|z|=60$ and $10 \%$ for $|g|=2 g_{D}$. For HIPs with $|\eta|<$ 1.375 that pass the level-1 trigger, the efficiency to satisfy the HLT and the remaining offline selection, described below, is generally $25 \%$ to $60 \%$.

Any data that fail the electron-photon data quality requirements are discarded as are events flagged as containing noise in the LAr calorimeter. The event selection then starts by identifying events containing at least one candidate featuring a topological cluster of EM calorimeter cells [55] with $E_{T}>18 \mathrm{GeV}$ in the $|\eta|<1.375$ region. The remaining selection is based on two powerful backgrounddiscriminating variables, denoted $f_{\mathrm{HT}}$ and $w$. The selected EM cluster candidates are used to seed the $f_{\mathrm{HT}}$ variable, which is similar to the trigger variable $f_{\mathrm{HT} \text {,trig }}$ except that an 8 -mm-wide rectangular road is used instead of a 10-mrad wedge, to better confine the hit counting to the region closest to the HIP trajectory. The $w$ variable gives a measure of the lateral energy dispersion of the EM cluster candidate. For each EM cluster candidate, the associated energy contained in the presampler, EM1 layer and EM2 layer is denoted by $E_{0}, E_{1}$, and $E_{2}$, respectively. Three $w_{i}$ variables $(i=0,1,2)$ are defined as the fraction of the EM cluster energy $E_{i}$ contained in the two most energetic cells in the presampler, the four most energetic cells in EM1, and the five most energetic cells in EM2, respectively. If the cluster energy in layer $E_{i}$ is confined to a single cell, then $w_{i}=1$, consistent with the narrow shower expected for HIPs. In each layer, the number of cells is chosen to optimize the signal efficiency and the discrimination power between HIPs and electron or jet backgrounds. The combined lateral energy dispersion $w$ is thus defined as the average of all $w_{i}(i=0,1,2)$ for which $E_{i}$ exceeds $10 \mathrm{GeV}$ (relaxed to $5 \mathrm{GeV}$ for $i=2$ ). The latter requirement ensures that only layers with energy deposits significantly above the cluster-level noise, which depends on both the cell-level noise and the cell granularity, contribute to the $w$ computation. In addition, at least one of the $E_{0}$ and $E_{1}$ requirements must be satisfied. The final selection requirements are $f_{\mathrm{HT}} \geq 0.7$ and $w \geq 0.96$, a choice which maximizes the signal-to-background ratio for the majority of the signal samples.

Backgrounds are random combinations of rare processes and need to be estimated directly from the collected data. Examples of background processes that could yield high $f_{\text {HT }}$ values include overlapping charged particles and noise in TRT straws. Processes that could yield high $w$ values include high-energy electrons and noise in EM calorimeter cells. The background estimation method relies on the fact that, in the background near the signal region, $f_{\mathrm{HT}}$ and $w$ are largely uncorrelated. Control regions $B, C$, and $D$ are defined as sidebands in $f_{\mathrm{HT}}$ and $w$ near the signal region $A$, as shown in Fig. 1. Region $A$ contains $90 \%$ or more of the signal for masses below $4000 \mathrm{GeV}$ for all simulated charges except $|z|=20$, where the fraction is $70 \%$. The numbers of events observed in the control regions are $N_{B}=1528$, 


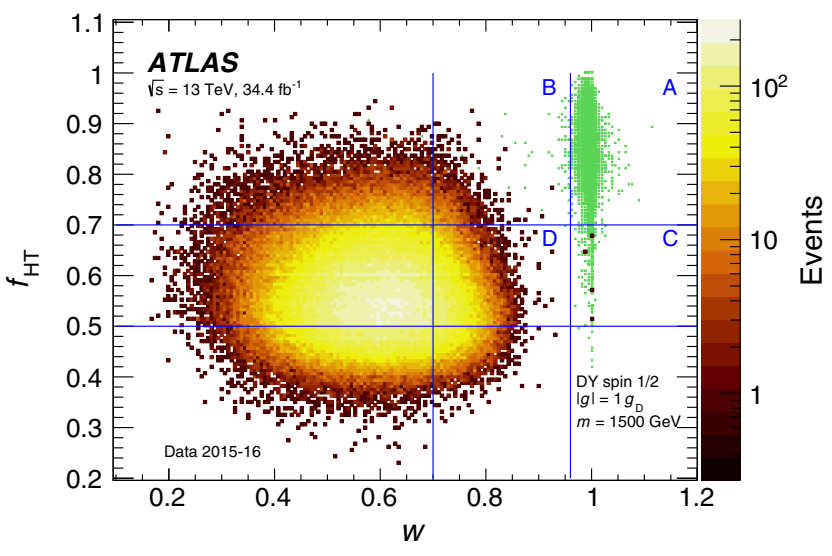

FIG. 1. Two-dimensional distribution of the two discriminating variables $f_{\mathrm{HT}}$ vs $w$ in data (color scale) and a typical HIP signal (green squares). The regions for defining the signal $(A)$ and for the background estimate and background validation $(B, D$, and C) are indicated.

$N_{C}=4$, and $N_{D}=30375$, and the expected background is calculated as $N_{A}^{\exp }=N_{B} N_{C} / N_{D}=0.20 \pm 0.11$ (stat) \pm 0.40 (syst). The latter uncertainty accounts for the fact that $f_{\mathrm{HT}}$ and $w$ each depend on $\eta$ but in different ways, resulting
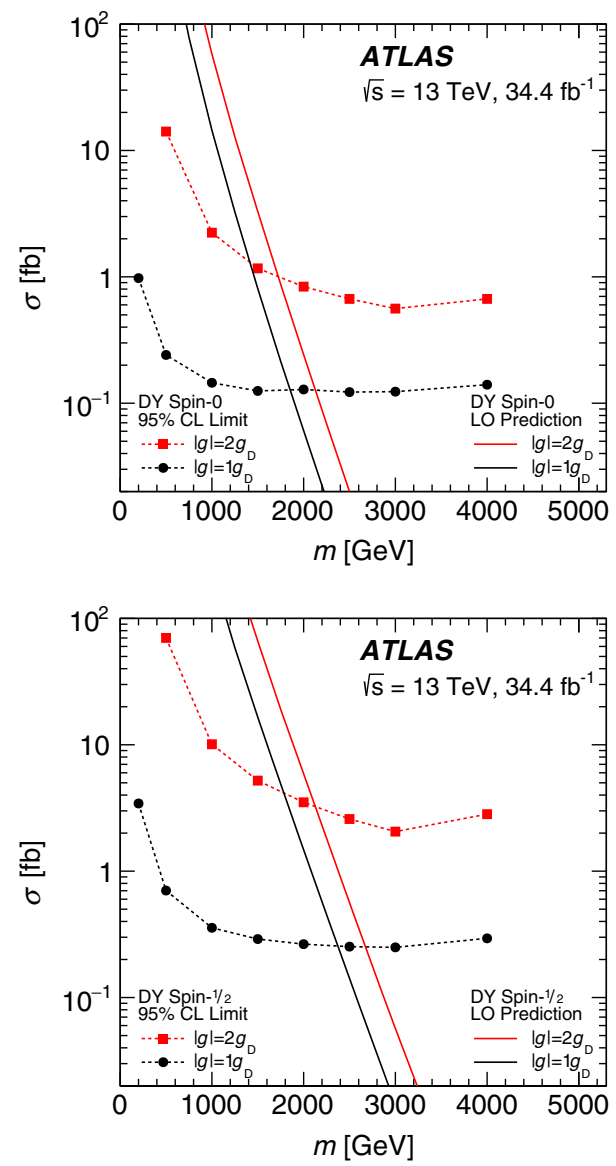

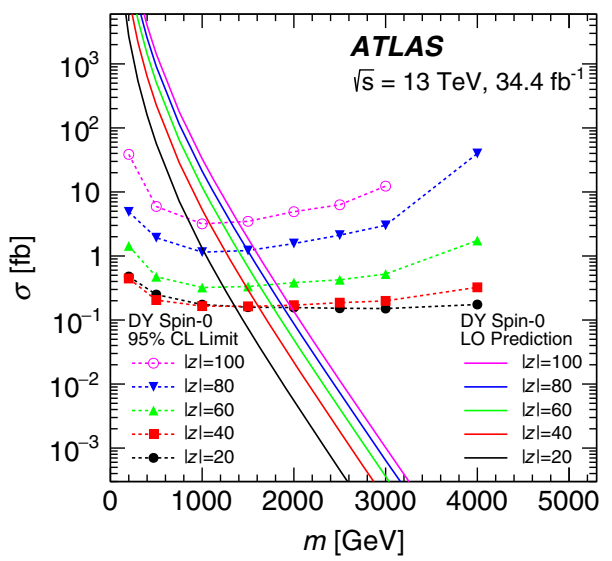

in a Pearson correlation coefficient of $10 \%$. This uncertainty was obtained by binning the $f_{\mathrm{HT}}-w$ plane into 0.025 -unit regions in $\eta$ and determining the maximum variation of the ratio of the numbers of events in the $B$ and $D$ regions. Simultaneous fits taking possible signal yields into account confirm that signal leakage into the $B$ and $\mathrm{C}$ control regions cannot significantly affect the background estimate. As an additional cross-check, the $B, C$, and $D$ regions are divided into various subregions, within which the background estimation is again performed. The estimated and observed event yields are consistent in all cases.

No event was observed in the signal region $A$ in $34.4 \mathrm{fb}^{-1}$ of $13 \mathrm{TeV} p p$ collision data, consistent with the background expectation. Thus, $95 \%$ confidence-level (C.L.) upper limits can be set on production cross sections for various signal hypotheses, using estimates of efficiencies and their corresponding uncertainties for each HIP charge, mass, and spin, as well as the uncertainty in the integrated luminosity $(2.2 \%$, estimated following the methods discussed in Ref. [56]). A C.L.s [57] frequentist framework implemented in RooStats [58] is used for hypothesis testing and to calculate confidence intervals. The resulting limits are shown as a function of HIP mass in Fig. 2 for monopoles and HECOs in

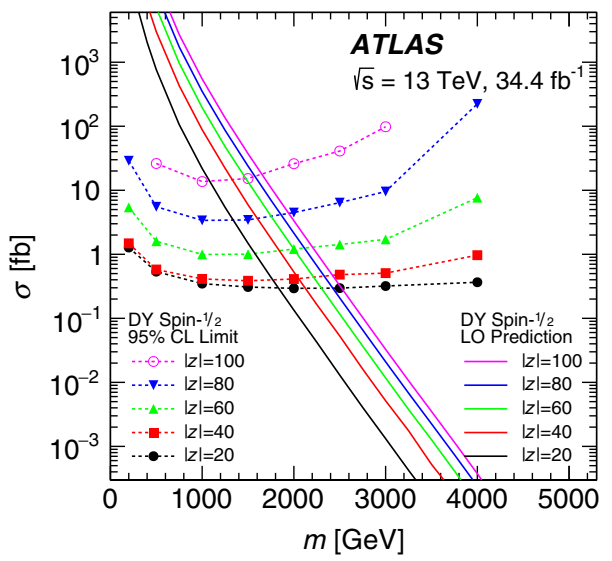

FIG. 2. Observed 95\% confidence-level upper limits on the cross section for Drell-Yan spin-0 (top) and spin-1/2 (bottom) monopole (left) and HECO (right) production as a function of mass (dashed lines with markers). The limits for spin-0 HIPs are more stringent than for spin- $1 / 2$ due to the higher selection efficiencies of the former. The theoretical leading-order cross sections are overlaid (solid lines). 
TABLE I. Lower limits on the mass of magnetic monopoles and HECOs (in $\mathrm{GeV}$ ) at $95 \%$ confidence level in models of spin-0 and spin-1/2 leading-order DY HIP pair production.

\begin{tabular}{lccccccc}
\hline \hline & \multicolumn{5}{c}{ Lower limits on the mass of Drell-Yan magnetic monopoles and HECOs [GeV]. } \\
\cline { 2 - 8 } & $|g|=1 g_{D}$ & $|g|=2 g_{D}$ & $|z|=20$ & $|z|=40$ & $|z|=60$ & $|z|=80$ & $|z|=100$ \\
\hline Spin-0 & 1850 & 1725 & 1355 & 1615 & 1625 & 1495 & 1390 \\
Spin-1/2 & 2370 & 2125 & 1830 & 2050 & 2000 & 1860 & 1650 \\
\hline \hline
\end{tabular}

the charge ranges where the search is sensitive for DY production with different spins. The mass dependence of the cross-section limits arises from a variation of the efficiencies with the HIP kinetic energy. The cross-section limits are relatively insensitive to the systematic uncertainties, which introduce variations no larger than $12 \%$ across all masscharge-spin points. Model cross-section predictions are shown in Fig. 2 as solid lines. The corresponding mass limits are shown in Table I. Given the uncertainty in the predicted cross sections, these mass limits primarily serve as benchmarks for comparison with other experiments. The MoEDAL experiment [31] is able to set slightly stronger monopole mass limits because they consider photon-fusion pair production in addition to the Drell-Yan mechanism. For most masses, the present cross-section limits obtained for magnetic charge $|g|=2 g_{D}$ surpass by 1 to 2 orders of magnitude the best previous constraints, also set by MoEDAL [31]. The cross-section limits obtained for HECOs and for monopoles with $|g|=g_{\mathrm{D}}$ surpass by approximately a factor of 5 the best constraints, set by the previous ATLAS analysis [27], and access the range $60<|z| \leq 100$ for the first time.

We thank CERN for the very successful operation of the LHC, as well as the support staff from our institutions without whom ATLAS could not be operated efficiently. We acknowledge the support of ANPCyT, Argentina; YerPhI, Armenia; ARC, Australia; FWF, BMWFW, Austria; ANAS, Azerbaijan; SSTC, Belarus; CNPq, FAPESP, Brazil; NSERC, CFI, NRC, Canada; CERN; CONICYT, Chile; CAS, NSFC, MOST, China; COLCIENCIAS, Colombia; VSC CR, MSMT CR, MPO CR, Czech Republic; DNSRC, DNRF, Denmark; IN2P3-CNRS, CEA-DRF/IRFU, France; SRNSFG, Georgia; MPG, HGF, BMBF, Germany; GSRT, Greece; RGC, Hong Kong SAR, Hong Kong China; Benoziyo Center, ISF, Israel; INFN, Italy; JSPS, MEXT, Japan; JINR; CNRST, Morocco; NWO, Netherlands; RCN, Norway; MNiSW, NCN, Poland; FCT, Portugal; MNE/IFA, Romania; NRC KI, MES of Russia, Russia Federation; MESTD, Serbia; MSSR, Slovakia; ARRS, MIZŠ, Slovenia; DST/NRF, South Africa; MINECO, Spain; SRC, Wallenberg Foundation, Sweden; Cantons of Bern and Geneva, SNSF, SERI, Switzerland; MOST, Taiwan; TAEK, Turkey; STFC, United Kingdom; DOE, NSF, United States of America. In addition, individual groups and members have received support from CRC, Compute Canada, Canarie, BCKDF, Canada; Marie
Skłodowska-Curie, COST, ERDF, ERC, Horizon 2020, European Union; ANR, Investissements d'Avenir Labex and Idex, France; AvH, DFG, Germany; Herakleitos, Thales and Aristeia programmes co-financed by EU-ESF and the Greek NSRF, Greece; BSF-NSF, GIF, Israel; PROMETEO Programme Generalitat Valenciana, CERCA Generalitat de Catalunya, Spain; Leverhulme Trust, The Royal Society, United Kingdom. The crucial computing support from all WLCG partners is acknowledged gratefully, in particular from CERN, the ATLAS Tier-1 facilities at TRIUMF (Canada), NDGF (Denmark, Norway, Sweden), CC-IN2P3 (France), KIT/GridKA (Germany), INFN-CNAF (Italy), NL-T1 (Netherlands), PIC (Spain), ASGC (Taiwan), RAL (UK) and BNL (USA), the Tier-2 facilities worldwide and large non-WLCG resource providers. Major contributors of computing resources are listed in Ref. [59].

[1] P. A. M. Dirac, Quantised singularities in the electromagnetic field, Proc. R. Soc. A 133, 60 (1931).

[2] P. A. M. Dirac, The theory of magnetic poles, Phys. Rev. 74, 817 (1948).

[3] G. 't Hooft, Magnetic monopoles in unified gauge theories, Nucl. Phys. B79, 276 (1974).

[4] A. M. Polyakov, Particle spectrum in the quantum field theory, Pisma Zh. Eksp. Teor. Fiz. 20, 430 (1974) [Particle spectrum in the quantum field theory, JETP Lett. 20, 194 (1974).]

[5] Y. M. Cho and D. Maison, Monopole configuration in Weinberg-Salam Model, Phys. Lett. B 391, 360 (1997).

[6] K. Kimm, J.H. Yoon, and Y.M. Cho, Finite energy electroweak dyon, Eur. Phys. J. C 75, 67 (2015).

[7] J. Ellis, N. E. Mavromatos, and T. You, The price of an electroweak monopole, Phys. Lett. B 756, 29 (2016).

[8] S. Arunasalam and A. Kobakhidze, Electroweak monopoles and the electroweak phase transition, Eur. Phys. J. C 77, 444 (2017).

[9] J. Ellis, N. E. Mavromatos, and T. You, Light-by-Light Scattering Constraint on Born-Infeld Theory, Phys. Rev. Lett. 118, 261802 (2017).

[10] T. W. B. Kibble, Topology of cosmic domains and strings, J. Phys. A 9, 1387 (1976).

[11] J. P. Preskill, Cosmological Production of Superheavy Magnetic Monopoles, Phys. Rev. Lett. 43, 1365 (1979).

[12] O. Gould and A. Rajantie, Magnetic Monopole Mass Bounds from Heavy-Ion Collisions and Neutron Stars, Phys. Rev. Lett. 119, 241601 (2017). 
[13] B. Holdom, J. Ren, and C. Zhang, Quark Matter May Not Be Strange, Phys. Rev. Lett. 120, 222001 (2018).

[14] E. Farhi and R. L. Jaffe, Strange matter, Phys. Rev. D 30, 2379 (1984).

[15] S. Coleman, Q-balls, Nucl. Phys. B262, 263 (1985).

[16] A. Kusenko and M. E. Shaposhnikov, Supersymmetric Q-balls as dark matter, Phys. Lett. B 418, 46 (1998).

[17] B. Koch, M. Bleicher, and H. Stöcker, Black holes at LHC?, J. Phys. G 34, S535 (2007).

[18] S. Burdin, M. Fairbairn, P. Mermod, D. Milstead, J. Pinfold, T. Sloan, and W. Taylor, Non-collider searches for stable massive particles, Phys. Rep. 582, 1 (2015).

[19] IceCube Collaboration, Searches for relativistic magnetic monopoles in IceCube, Eur. Phys. J. C 76, 133 (2016).

[20] M. Fairbairn, A. C. Kraan, D. A. Milstead, T. Sjöstrand, P. Skands, and T. Sloan, Stable massive particles at colliders, Phys. Rep. 438, 1 (2007).

[21] CDF Collaboration, Direct Search for Dirac Magnetic Monopoles in $p \bar{p}$ Collisions at $\sqrt{s}=1.96 \mathrm{TeV}$, Phys. Rev. Lett. 96, 201801 (2006).

[22] STAR Collaboration, Strangelet search in $\mathrm{Au}+\mathrm{Au}$ collisions at $\sqrt{s_{N N}}=200 \mathrm{GeV}$, Phys. Rev. C 76, 011901 (2007).

[23] OPAL Collaboration, Search for Dirac magnetic monopoles in $e^{+} e^{-}$collisions with the OPAL detector at LEP2, Phys. Lett. B 663, 37 (2008).

[24] ATLAS Collaboration, Search for massive long-lived highly ionising particles with the ATLAS detector at the LHC, Phys. Lett. B 698, 353 (2011).

[25] ATLAS Collaboration, Search for Magnetic Monopoles in $\sqrt{s}=7 \mathrm{TeV} p p$ Collisions with the ATLAS Detector, Phys. Rev. Lett. 109, 261803 (2012).

[26] ATLAS Collaboration, Search for long-lived, multi-charged particles in $p p$ collisions at $\sqrt{s}=7 \mathrm{TeV}$ using the ATLAS detector, Phys. Lett. B 722, 305 (2013).

[27] ATLAS Collaboration, Search for magnetic monopoles and stable particles with high electric charges in $8 \mathrm{TeV} p p$ collisions with the ATLAS detector, Phys. Rev. D 93, 052009 (2016).

[28] MoEDAL Collaboration, Search for magnetic monopoles with the MoEDAL prototype trapping detector in $8 \mathrm{TeV}$ proton-proton collisions at the LHC, J. High Energy Phys. 08 (2016) 067.

[29] MoEDAL Collaboration, Search for Magnetic Monopoles with the MoEDAL Forward Trapping Detector in $13 \mathrm{TeV}$ Proton-Proton Collisions at the LHC, Phys. Rev. Lett. 118, 061801 (2017).

[30] MoEDAL Collaboration, Search for magnetic monopoles with the MoEDAL forward trapping detector in $2.11 \mathrm{fb}^{-1}$ of $13 \mathrm{TeV}$ proton-proton collisions at the LHC, Phys. Lett. B 782, 510 (2018).

[31] MoEDAL Collaboration, Magnetic Monopole Search with the Full MoEDAL Trapping Detector in $13 \mathrm{TeV}$ pp Collisions Interpreted in Photon-Fusion and Drell-Yan Production, Phys. Rev. Lett. 123, 021802 (2019).

[32] ATLAS Collaboration, Search for heavy long-lived multicharged particles in $p p$ collisions at $\sqrt{s}=8 \mathrm{TeV}$ using the ATLAS detector, Eur. Phys. J. C 75, 362 (2015).
[33] CMS Collaboration, Searches for long-lived charged particles in pp collisions at $\sqrt{s}=7$ and $8 \mathrm{TeV}$, J. High Energy Phys. 07 (2013) 122.

[34] ATLAS Collaboration, Search for heavy long-lived multicharged particles in proton-proton collisions at $\sqrt{s}=$ $13 \mathrm{TeV}$ using the ATLAS detector, Phys. Rev. D 99, 052003 (2019).

[35] J. S. Schwinger, Magnetic charge and quantum field theory, Phys. Rev. 144, 1087 (1966).

[36] J. S. Schwinger, Sources and magnetic charge, Phys. Rev. 173, 1536 (1968).

[37] J. S. Schwinger, A magnetic model of matter, Science 165, 757 (1969).

[38] A. De Roeck, A. Katre, P. Mermod, D. Milstead, and T. Sloan, Sensitivity of LHC experiments to exotic highly ionising particles, Eur. Phys. J. C 72, 1985 (2012).

[39] ATLAS Collaboration, The ATLAS experiment at the CERN Large Hadron Collider, J. Instrum. 3, S08003 (2008).

[40] S. Agostinelli et al., Geant4-A simulation toolkit, Nucl. Instrum. Methods Phys. Res., Sect. A 506, 250 (2003).

[41] ATLAS Collaboration, The ATLAS simulation infrastructure, Eur. Phys. J. C 70, 823 (2010).

[42] S. P. Ahlen, Stopping-power formula for magnetic monopoles, Phys. Rev. D 17, 229 (1978).

[43] S.P. Ahlen, Theoretical and experimental aspects of the energy loss of relativistic heavily ionizing particles, Rev. Mod. Phys. 52, 121 (1980).

[44] S. P. Ahlen and K. Kinoshita, Calculation of the stopping power of very-low-velocity magnetic monopoles, Phys. Rev. D 26, 2347 (1982).

[45] S. Cecchini, L. Patrizii, Z. Sahnoun, G. Sirri, and V. Togo, Energy losses of magnetic monopoles in aluminum, iron and copper, arXiv:1606.01220.

[46] S. Burdin, M. Horbatsch, and W. Taylor, A correction to Birks' Law in liquid argon ionization chamber simulations for highly ionizing particles, Nucl. Instrum. Methods Phys. Res., Sect. A 664, 111 (2012).

[47] J. Alwall, R. Frederix, S. Frixione, V. Hirschi, F. Maltoni, O. Mattelaer, H.-S. Shao, T. Stelzer, P. Torrielli, and M. Zaro, The automated computation of tree-level and next-toleading order differential cross sections, and their matching to parton shower simulations, J. High Energy Phys. 07 (2014) 079.

[48] K. Milton, Theoretical and experimental status of magnetic monopoles, Rep. Prog. Phys. 69, 1637 (2006).

[49] T. Sjöstrand, S. Mrenna, and P. Skands, PYTHIA 6.4 physics and manual, J. High Energy Phys. 05 (2006) 026.

[50] T. Sjöstrand, S. Mrenna, and P. Skands, A brief introduction to PYTHIA 8.1, Comput. Phys. Commun. 178, 852 (2008).

[51] NNPDF Collaboration, Parton distributions with LHC data, Nucl. Phys. B867, 244 (2013).

[52] ATLAS Collaboration, ATLAS Pythia 8 tunes to $7 \mathrm{TeV}$ data, ATL-PHYS-PUB-2014-021, 2014, https://cds.cern.ch/ record/1966419.

[53] ATLAS Collaboration, Summary of ATLAS Pythia 8 tunes, ATL-PHYS-PUB-2012-003, 2012, https://cds.cern.ch/ record/1474107. 
[54] ATLAS Collaboration, Performance of the ATLAS trigger system in 2015, Eur. Phys. J. C 77, 317 (2017).

[55] ATLAS Collaboration, Topological cell clustering in the ATLAS calorimeters and its performance in LHC Run 1, Eur. Phys. J. C 77, 490 (2017).

[56] ATLAS Collaboration, Luminosity determination in $p p$ collisions at $\sqrt{s}=8 \mathrm{TeV}$ using the ATLAS detector at the LHC, Eur. Phys. J. C 76, 653 (2016).
[57] A. L. Read, Presentation of search results: The CLs technique, J. Phys. G 28, 2693 (2002).

[58] W. Verkerke and D. Kirkby, The RooFit toolkit for data modeling, Conference in Computing in High Energy and Nuclear Physics (CHEP03) (2003), arXiv:physics/0306116.

[59] ATLAS Collaboration, ATLAS Computing Acknowledgements, ATL-GEN-PUB-2016-002, https://cds.cern.ch/ record/2202407.

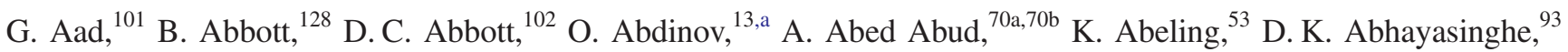
S. H. Abidi, ${ }^{167}$ O. S. AbouZeid, ${ }^{40}$ N. L. Abraham, ${ }^{156}$ H. Abramowicz, ${ }^{161}$ H. Abreu, ${ }^{160}$ Y. Abulaiti, ${ }^{6}$ B. S. Acharya, ${ }^{66 a, 66 b, b}$ B. Achkar, ${ }^{53}$ S. Adachi, ${ }^{163}$ L. Adam, ${ }^{99}$ C. Adam Bourdarios, ${ }^{132}$ L. Adamczyk, ${ }^{83 a}$ L. Adamek, ${ }^{167}$ J. Adelman, ${ }^{121}$ M. Adersberger ${ }^{114}$ A. Adiguzel, ${ }^{12 c, c}$ S. Adorni,${ }^{54}$ T. Adye, ${ }^{144}$ A. A. Affolder, ${ }^{146}$ Y. Afik, ${ }^{160}$ C. Agapopoulou, ${ }^{132}$ M. N. Agaras, ${ }^{38}$ A. Aggarwal, ${ }^{119}$ C. Agheorghiesei, ${ }^{27 \mathrm{c}}$ J. A. Aguilar-Saavedra, ${ }^{140 \mathrm{f}, 140 \mathrm{a}, \mathrm{d}}$ F. Ahmadov, ${ }^{79}$ W. S. Ahmed, ${ }^{103}$ X. Ai, ${ }^{15 a}$ G. Aielli, ${ }^{73 a, 73 b}$ S. Akatsuka, ${ }^{85}$ T. P. A. Åkesson, ${ }^{96}$ E. Akilli, ${ }^{54}$ A. V. Akimov, ${ }^{110}$ K. Al Khoury, ${ }^{132}$ G. L. Alberghi, ${ }^{23 b, 23 a}$ J. Albert, ${ }^{176}$ M. J. Alconada Verzini, ${ }^{88}$ S. Alderweireldt, ${ }^{36}$ M. Aleksa, ${ }^{36}$ I. N. Aleksandrov, ${ }^{79}$ C. Alexa, ${ }^{27 b}$ D. Alexandre, ${ }^{19}$ T. Alexopoulos, ${ }^{10}$ A. Alfonsi, ${ }^{120}$ M. Alhroob, ${ }^{128}$ B. Ali, ${ }^{142}$ G. Alimonti, ${ }^{68 a}$ J. Alison, ${ }^{37}$ S. P. Alkire, ${ }^{148}$ C. Allaire, ${ }^{132}$ B. M. M. Allbrooke, ${ }^{156}$ B. W. Allen, ${ }^{131}$ P. P. Allport, ${ }^{21}$ A. Aloisio, ${ }^{69 a, 69 b}$ A. Alonso, ${ }^{40}$ F. Alonso, ${ }^{88}$ C. Alpigiani, ${ }^{148}$ A. A. Alshehri, ${ }^{57}$ M. Alvarez Estevez, ${ }^{98}$ D. Álvarez Piqueras, ${ }^{174}$ M. G. Alviggi ${ }^{69 a, 69 b}$ Y. Amaral Coutinho, ${ }^{80 b}$ A. Ambler, ${ }^{103}$ L. Ambroz,${ }^{135}$ C. Amelung, ${ }^{26}$ D. Amidei, ${ }^{105}$ S. P. Amor Dos Santos, ${ }^{140 a}$ S. Amoroso, ${ }^{46}$ C. S. Amrouche, ${ }^{54}$ F. An,${ }^{78}$ C. Anastopoulos, ${ }^{149}$ N. Andari, ${ }^{145}$ T. Andeen, ${ }^{11}$ C. F. Anders,,${ }^{61 b}$ J. K. Anders, ${ }^{20}$ A. Andreazza,${ }^{6 a, 68 b}$ V. Andrei, ${ }^{61 a}$ C. R. Anelli, ${ }^{176}$ S. Angelidakis, ${ }^{38}$ A. Angerami, ${ }^{39}$ A. V. Anisenkov, ${ }^{122 b, 122 a}$ A. Annovi, ${ }^{71 a}$ C. Antel, ${ }^{61 \mathrm{a}}$ M. T. Anthony, ${ }^{149}$ M. Antonelli, ${ }^{51}$ D. J. A. Antrim, ${ }^{171}$ F. Anulli, ${ }^{72 a}$ M. Aoki,${ }^{81}$ J. A. Aparisi Pozo, ${ }^{174}$ L. Aperio Bella, ${ }^{36}$ G. Arabidze, ${ }^{106}$ J. P. Araque, ${ }^{140 \mathrm{a}}$ V. Araujo Ferraz, ${ }^{80 \mathrm{~b}}$ R. Araujo Pereira, ${ }^{80 \mathrm{~b}}$ C. Arcangeletti, ${ }^{51}$ A. T. H. Arce, ${ }^{49}$ F. A. Arduh, ${ }^{88}$ J-F. Arguin, ${ }^{109}$ S. Argyropoulos, ${ }^{77}$ J.-H. Arling, ${ }^{46}$ A. J. Armbruster, ${ }^{36}$ L. J. Armitage, ${ }^{92}$ A. Armstrong, ${ }^{171}$ O. Arnaez, ${ }^{167}$ H. Arnold, ${ }^{120}$ A. Artamonov, ${ }^{111, a}$ G. Artoni, ${ }^{135}$ S. Artz, ${ }^{99}$ S. Asai, ${ }^{163}$ N. Asbah, ${ }^{59}$ E. M. Asimakopoulou, ${ }^{172}$ L. Asquith, ${ }^{156}$ K. Assamagan, ${ }^{29}$ R. Astalos, ${ }^{28 a}$ R. J. Atkin, ${ }^{33 a}$ M. Atkinson, ${ }^{173}$ N. B. Atlay, ${ }^{151}$ H. Atmani, ${ }^{132}$ K. Augsten, ${ }^{142}$ G. Avolio, ${ }^{36}$ R. Avramidou, ${ }^{60 a}$ M. K. Ayoub,${ }^{15 a}$ A. M. Azoulay, ${ }^{168 b}$ G. Azuelos, ${ }^{109, e}$ M. J. Baca, ${ }^{21}$ H. Bachacou, ${ }^{145}$ K. Bachas, ${ }^{67 a, 67 b}$ M. Backes, ${ }^{135}$ F. Backman, ${ }^{45 a, 45 b}$ P. Bagnaia, ${ }^{72 a, 72 b}$ M. Bahmani, ${ }^{84}$ H. Bahrasemani, ${ }^{152}$ A. J. Bailey, ${ }^{174}$ V. R. Bailey, ${ }^{173}$ J. T. Baines, ${ }^{144}$ M. Bajic, ${ }^{40}$ C. Bakalis, ${ }^{10}$ O. K. Baker, ${ }^{183}$ P. J. Bakker, ${ }^{120}$ D. Bakshi Gupta, ${ }^{8}$ S. Balaji, ${ }^{157}$ E. M. Baldin, ${ }^{122 b, 122 a}$ P. Balek, ${ }^{180}$ F. Balli, ${ }^{145}$ W. K. Balunas, ${ }^{135}$ J. Balz, ${ }^{99}$ E. Banas, ${ }^{84}$ A. Bandyopadhyay, ${ }^{24}$ Sw. Banerjee, ${ }^{181, f}$ A. A. E. Bannoura, ${ }^{182}$ L. Barak,${ }^{161}$ W. M. Barbe,${ }^{38}$ E. L. Barberio, ${ }^{104}$ D. Barberis, ${ }^{55 b, 55 a}$ M. Barbero, ${ }^{101}$ T. Barillari, ${ }^{115} \mathrm{M}-\mathrm{S}$. Barisits, ${ }^{36}$ J. Barkeloo, ${ }^{131}$ T. Barklow, ${ }^{153}$ R. Barnea, ${ }^{160}$ S. L. Barnes, ${ }^{60 \mathrm{c}}$ B. M. Barnett, ${ }^{144}$ R. M. Barnett, ${ }^{18}$ Z. Barnovska-Blenessy, ${ }^{60 a}$ A. Baroncelli, ${ }^{60 a}$ G. Barone, ${ }^{29}$ A. J. Barr, ${ }^{135}$ L. Barranco Navarro, ${ }^{45 a, 45 b}$ F. Barreiro, ${ }^{98}$ J. Barreiro Guimarães da Costa, ${ }^{15 a}$ S. Barsov,${ }^{138}$ R. Bartoldus, ${ }^{153}$ G. Bartolini, ${ }^{101}$ A. E. Barton ${ }^{89}$ P. Bartos, ${ }^{28 a}$ A. Basalaev, ${ }^{46}$ A. Bassalat, ${ }^{132, g}$ R. L. Bates ${ }^{57}$ S. J. Batista, ${ }^{167}$ S. Batlamous, ${ }^{35 \mathrm{e}}$ J. R. Batley, ${ }^{32}$ B. Batool ${ }^{151}$ M. Battaglia, ${ }^{146}$ M. Bauce, ${ }^{72 a, 72 b}$ F. Bauer ${ }^{145}$ K. T. Bauer,${ }^{171}$ H. S. Bawa, ${ }^{31, h}$ J. B. Beacham,${ }^{49}$ T. Beau, ${ }^{136}$ P. H. Beauchemin, ${ }^{170}$ F. Becherer, ${ }^{52}$ P. Bechtle, ${ }^{24}$ H. C. Beck, ${ }^{53}$ H. P. Beck ${ }^{20, i}$ K. Becker,${ }^{52}$ M. Becker, ${ }^{99}$ C. Becot, ${ }^{46}$ A. Beddall, ${ }^{12 \mathrm{~d}}$ A. J. Beddall, ${ }^{12 \mathrm{a}}$ V. A. Bednyakov, ${ }^{79}$ M. Bedognetti, ${ }^{120}$ C. P. Bee, ${ }^{155}$ T. A. Beermann, ${ }^{76}$ M. Begalli, ${ }^{80 \mathrm{~b}}$ M. Begel,${ }^{29}$ A. Behera, ${ }^{155}$ J. K. Behr,${ }^{46}$ F. Beisiegel,${ }^{24}$ A. S. Bell, ${ }^{94}$ G. Bella, ${ }^{161}$ L. Bellagamba, ${ }^{23 b}$ A. Bellerive, ${ }^{34}$ P. Bellos, ${ }^{9}$ K. Beloborodov, ${ }^{122 b, 122 a}$ K. Belotskiy, ${ }^{112}$ N. L. Belyaev, ${ }^{112}$ D. Benchekroun, ${ }^{35 a}$ N. Benekos, ${ }^{10}$ Y. Benhammou, ${ }^{161}$

D. P. Benjamin, ${ }^{6}$ M. Benoit, ${ }^{54}$ J. R. Bensinger, ${ }^{26}$ S. Bentvelsen, ${ }^{120}$ L. Beresford, ${ }^{135}$ M. Beretta, ${ }^{51}$ D. Berge,${ }^{46}$ E. Bergeaas Kuutmann, ${ }^{172}$ N. Berger, ${ }^{5}$ B. Bergmann, ${ }^{142}$ L. J. Bergsten, ${ }^{26}$ J. Beringer, ${ }^{18}$ S. Berlendis, ${ }^{7}$ N. R. Bernard, ${ }^{102}$ G. Bernardi, ${ }^{136}$ C. Bernius, ${ }^{153}$ T. Berry, ${ }^{93}$ P. Berta,${ }^{99}$ C. Bertella, ${ }^{15 a}$ I. A. Bertram, ${ }^{89}$ G. J. Besjes, ${ }^{40}$ O. Bessidskaia Bylund, ${ }^{182}$ N. Besson, ${ }^{145}$ A. Bethani, ${ }^{100}$ S. Bethke, ${ }^{115}$ A. Betti, ${ }^{24}$ A. J. Bevan, ${ }^{92}$ J. Beyer, ${ }^{115}$ R. Bi, ${ }^{139}$ R. M. Bianchi, ${ }^{139}$ O. Biebel,,${ }^{114}$ D. Biedermann, ${ }^{19}$ R. Bielski, ${ }^{36}$ K. Bierwagen, ${ }^{99}$ N. V. Biesuz,${ }^{71 a, 71 b}$ M. Biglietti ${ }^{74 a}$ T. R. V. Billoud, ${ }^{109}$ M. Bindi, ${ }^{53}$ A. Bingul, ${ }^{12 \mathrm{~d}}$ C. Bini,${ }^{72 \mathrm{a}, 72 \mathrm{~b}}$ S. Biondi, ${ }^{23 \mathrm{~b}, 23 \mathrm{a}}$ M. Birman, ${ }^{180}$ T. Bisanz, ${ }^{53}$ J. P. Biswal,${ }^{161}$ A. Bitadze,${ }^{100}$ C. Bittrich, ${ }^{48}$ K. Bjørke, ${ }^{134}$ K. M. Black, ${ }^{25}$ T. Blazek, ${ }^{28 a}$ I. Bloch, ${ }^{46}$ C. Blocker, ${ }^{26}$ A. Blue, ${ }^{57}$ U. Blumenschein, ${ }^{92}$ G. J. Bobbink, ${ }^{120}$ 
V. S. Bobrovnikov, ${ }^{122 b, 122 a}$ S. S. Bocchetta, ${ }^{96}$ A. Bocci ${ }^{49}$ D. Boerner, ${ }^{46}$ D. Bogavac, ${ }^{14}$ A. G. Bogdanchikov, ${ }^{122 b, 122 a}$

C. Bohm, ${ }^{45 a}$ V. Boisvert, ${ }^{93}$ P. Bokan,${ }^{53,172}$ T. Bold ${ }^{83 a}$ A. S. Boldyrev, ${ }^{113}$ A. E. Bolz, ${ }^{61 b}$ M. Bomben, ${ }^{136}$ M. Bona, ${ }^{92}$ J. S. Bonilla, ${ }^{131}$ M. Boonekamp, ${ }^{145}$ H. M. Borecka-Bielska, ${ }^{90}$ A. Borisov, ${ }^{123}$ G. Borissov, ${ }^{89}$ J. Bortfeldt, ${ }^{36}$ D. Bortoletto, ${ }^{135}$

V. Bortolotto, ${ }^{73 a, 73 b}$ D. Boscherini, ${ }^{23 b}$ M. Bosman, ${ }^{14}$ J. D. Bossio Sola ${ }^{103}$ K. Bouaouda, ${ }^{35 a}$ J. Boudreau, ${ }^{139}$

E. V. Bouhova-Thacker, ${ }^{89}$ D. Boumediene, ${ }^{38}$ S. K. Boutle, ${ }^{57}$ A. Boveia, ${ }^{126}$ J. Boyd,${ }^{36}$ D. Boye,${ }^{33 b, j}$ I. R. Boyko, ${ }^{79}$ A. J. Bozson, ${ }^{93}$ J. Bracinik, ${ }^{21}$ N. Brahimi, ${ }^{101}$ G. Brandt, ${ }^{182}$ O. Brandt, ${ }^{61 a}$ F. Braren, ${ }^{46}$ U. Bratzler, ${ }^{164}$ B. Brau,${ }^{102}$ J. E. Brau, ${ }^{131}$ W. D. Breaden Madden ${ }^{57}$ K. Brendlinger ${ }^{46}$ L. Brenner, ${ }^{46}$ R. Brenner, ${ }^{172}$ S. Bressler, ${ }^{180}$ B. Brickwedde, ${ }^{99}$ D. L. Briglin, ${ }^{21}$ D. Britton, ${ }^{57}$ D. Britzger, ${ }^{15}$ I. Brock, ${ }^{24}$ R. Brock, ${ }^{106}$ G. Brooijmans, ${ }^{39}$ W. K. Brooks, ${ }^{147 b}$ E. Brost, ${ }^{121}$ J. H Broughton, ${ }^{21}$ P. A. Bruckman de Renstrom ${ }^{84}$ D. Bruncko, ${ }^{28 b}$ A. Bruni, ${ }^{23 b}$ G. Bruni, ${ }^{23 b}$ L. S. Bruni, ${ }^{120}$ S. Bruno, ${ }^{73 a, 73 b}$ B. H. Brunt, ${ }^{32}$ M. Bruschi, ${ }^{23 b}$ N. Bruscino, ${ }^{139}$ P. Bryant,${ }^{37}$ L. Bryngemark, ${ }^{96}$ T. Buanes,${ }^{17}$ Q. Buat,${ }^{36}$ P. Buchholz, ${ }^{151}$ A. G. Buckley, ${ }^{57}$ I. A. Budagov, ${ }^{79}$ M. K. Bugge, ${ }^{134}$ F. Bührer, ${ }^{52}$ O. Bulekov, ${ }^{112}$ T. J. Burch, ${ }^{121}$ S. Burdin, ${ }^{90}$ C. D. Burgard, ${ }^{120}$ A. M. Burger, ${ }^{129}$ B. Burghgrave, ${ }^{8}$ K. Burka, ${ }^{84}$ J. T. P. Burr, ${ }^{46}$ J. C. Burzynski, ${ }^{102}$ V. Büscher,${ }^{99}$ E. Buschmann,${ }^{53}$ P. J. Bussey, ${ }^{57}$ J. M. Butler, ${ }^{25}$ C. M. Buttar, ${ }^{57}$ J. M. Butterworth, ${ }^{94}$ P. Butti, ${ }^{36}$ W. Buttinger, ${ }^{36}$ A. Buzatu, ${ }^{158}$ A. R. Buzykaev, ${ }^{122 b, 122 a}$ G. Cabras, ${ }^{23 b, 23 a}$ S. Cabrera Urbán, ${ }^{174}$ D. Caforio, ${ }^{56}$ H. Cai, ${ }^{173}$ V. M. M. Cairo, ${ }^{153}$ O. Cakir, ${ }^{4 a}$ N. Calace, ${ }^{36}$ P. Calafiura, ${ }^{18}$ A. Calandri, ${ }^{101}$ G. Calderini, ${ }^{136}$ P. Calfayan, ${ }^{65}$ G. Callea, ${ }^{57}$ L. P. Caloba, ${ }^{80 b}$ S. Calvente Lopez, ${ }^{98}$ D. Calvet, ${ }^{38}$ S. Calvet, ${ }^{38}$ T. P. Calvet, ${ }^{155}$ M. Calvetti, ${ }^{71 \mathrm{a}, 71 \mathrm{~b}}$ R. Camacho Toro, ${ }^{136}$ S. Camarda, ${ }^{36}$ D. Camarero Munoz, ${ }^{98}$ P. Camarri, ${ }^{73 a, 73 \mathrm{~b}}$ D. Cameron, ${ }^{134}$ R. Caminal Armadans, ${ }^{102}$ C. Camincher ${ }^{36}$ S. Campana, ${ }^{36}$ M. Campanelli,${ }^{94}$ A. Camplani, ${ }^{40}$ A. Campoverde, ${ }^{151}$ V. Canale ${ }^{69 a, 69 b}$ A. Canesse, ${ }^{103}$ M. Cano Bret,${ }^{60 \mathrm{c}}$ J. Cantero, ${ }^{129}$ T. Cao, ${ }^{161}$ Y. Cao, ${ }^{173}$ M. D. M. Capeans Garrido, ${ }^{36}$ M. Capua, ${ }^{41 b, 41 a}$ R. Cardarelli, ${ }^{73 a}$ F. C. Cardillo, ${ }^{149}$ G. Carducci, ${ }^{41 b, 41 a}$ I. Carli, ${ }^{143}$ T. Carli, ${ }^{36}$ G. Carlino,${ }^{69 a}$ B. T. Carlson, ${ }^{139}$ L. Carminati, ${ }^{68 a, 68 b}$ R. M. D. Carney, ${ }^{45 a, 45 b}$ S. Caron, ${ }^{119}$ E. Carquin, ${ }^{147 b}$ S. Carrá, ${ }^{46}$ J. W. S. Carter, ${ }^{167}$ M. P. Casado, ${ }^{14, k}$ A. F. Casha, ${ }^{167}$ D. W. Casper, ${ }^{171}$ R. Castelijn, ${ }^{120}$ F. L. Castillo, ${ }^{174}$ V. Castillo Gimenez, ${ }^{174}$ N. F. Castro, ${ }^{140 a, 140 e}$ A. Catinaccio, ${ }^{36}$ J. R. Catmore, ${ }^{134}$ A. Cattai,${ }^{36}$ J. Caudron,${ }^{24}$ V. Cavaliere, ${ }^{29}$ E. Cavallaro, ${ }^{14}$ M. Cavalli-Sforza, ${ }^{14}$ V. Cavasinni, ${ }^{71 a, 71 b}$ E. Celebi, ${ }^{12 b}$ F. Ceradini, ${ }^{74 a, 74 b}$ L. Cerda Alberich, ${ }^{174}$ K. Cerny, ${ }^{130}$ A. S. Cerqueira ${ }^{80 a}$ A. Cerri, ${ }^{156}$ L. Cerrito, ${ }^{73 a, 73 b}$ F. Cerutti, ${ }^{18}$ A. Cervelli, ${ }^{23 b, 23 a}$ S. A. Cetin, ${ }^{12 b}$ D. Chakraborty, ${ }^{121}$ S. K. Chan, ${ }^{59}$ W. S. Chan, ${ }^{120}$ W. Y. Chan,${ }^{90}$ J. D. Chapman, ${ }^{32}$ B. Chargeishvili, ${ }^{159 b}$ D. G. Charlton, ${ }^{21}$ T. P. Charman, ${ }^{92}$ C. C. Chau, ${ }^{34}$ S. Che, ${ }^{126}$ A. Chegwidden, ${ }^{106}$ S. Chekanov, ${ }^{6}$ S. V. Chekulaev, ${ }^{168 a}$ G. A. Chelkov, ${ }^{79,1}$ M. A. Chelstowska, ${ }^{36}$ B. Chen,${ }^{78}$ C. Chen, ${ }^{60 a}$ C. H. Chen, ${ }^{78}$ H. Chen, ${ }^{29}$ J. Chen, ${ }^{60 \mathrm{a}}$ J. Chen,${ }^{39}$ S. Chen, ${ }^{137}$ S. J. Chen, ${ }^{15 \mathrm{c}}$ X. Chen, ${ }^{15 b, \mathrm{~m}}$ Y. Chen,${ }^{82}$ Y-H. Chen, ${ }^{46}$ H. C. Cheng, ${ }^{63 \mathrm{a}}$ H. J. Cheng, ${ }^{15 a, 15 \mathrm{~d}}$ A. Cheplakov, ${ }^{79}$ E. Cheremushkina, ${ }^{123}$ R. Cherkaoui El Moursli, ${ }^{35 e}$ E. Cheu, ${ }^{7}$ K. Cheung, ${ }^{64}$ T. J. A. Chevalérias, ${ }^{145}$ L. Chevalier, ${ }^{145}$ V. Chiarella, ${ }^{51}$ G. Chiarelli, ${ }^{71 a}$ G. Chiodini, ${ }^{67 a}$ A. S. Chisholm, ${ }^{36,21}$ A. Chitan, ${ }^{27 b}$ I. Chiu, ${ }^{163}$ Y. H. Chiu, ${ }^{176}$ M. V. Chizhov, ${ }^{79}$ K. Choi, ${ }^{65}$ A. R. Chomont, ${ }^{72 a, 72 b}$ S. Chouridou, ${ }^{162}$ Y. S. Chow, ${ }^{120}$ M. C. Chu, ${ }^{63 a}$ J. Chudoba, ${ }^{141}$ A. J. Chuinard, ${ }^{103}$ J. J. Chwastowski, ${ }^{84}$ L. Chytka, ${ }^{130}$ K. M. Ciesla,${ }^{84}$ D. Cinca,${ }^{47}$ V. Cindro, ${ }^{91}$ I. A. Cioară, ${ }^{27 b}$ A. Ciocio, ${ }^{18}$ F. Cirotto, ${ }^{69 a, 69 b}$ Z. H. Citron, ${ }^{180}$ M. Citterio, ${ }^{68 \mathrm{a}}$ D. A. Ciubotaru, ${ }^{27 b}$ B. M. Ciungu, ${ }^{167}$ A. Clark, ${ }^{54}$ M. R. Clark, ${ }^{39}$ P. J. Clark, ${ }^{50}$

C. Clement, ${ }^{45,45 b}$ Y. Coadou, ${ }^{101}$ M. Cobal, ${ }^{66 a, 66 c}$ A. Coccaro, ${ }^{55 b}$ J. Cochran, ${ }^{78}$ H. Cohen, ${ }^{161}$ A. E. C. Coimbra, ${ }^{36}$ L. Colasurdo, ${ }^{119}$ B. Cole, ${ }^{39}$ A. P. Colijn, ${ }^{120}$ J. Collot,${ }^{58}$ P. Conde Muiño, ${ }^{140 a, n}$ E. Coniavitis,${ }^{52}$ S. H. Connell, ${ }^{33 b}$ I. A. Connelly, ${ }^{57}$ S. Constantinescu, ${ }^{27 b}$ F. Conventi, ${ }^{69 a, 0}$ A. M. Cooper-Sarkar, ${ }^{135}$ F. Cormier,${ }^{175}$ K. J. R. Cormier, ${ }^{167}$ L. D. Corpe ${ }^{94}$ M. Corradi, ${ }^{72 a, 72 b}$ E. E. Corrigan, ${ }^{96}$ F. Corriveau, ${ }^{103, p}$ A. Cortes-Gonzalez, ${ }^{36}$ M. J. Costa, ${ }^{174}$ F. Costanza, ${ }^{5}$ D. Costanzo, ${ }^{149}$ G. Cowan, ${ }^{93}$ J. W. Cowley, ${ }^{32}$ J. Crane, ${ }^{100}$ K. Cranmer,${ }^{124}$ S. J. Crawley, ${ }^{57}$ R. A. Creager, ${ }^{137}$ S. Crépé-Renaudin, ${ }^{58}$ F. Crescioli, ${ }^{136}$ M. Cristinziani, ${ }^{24}$ V. Croft, ${ }^{120}$ G. Crosetti, ${ }^{41 b, 41 a}$ A. Cueto, ${ }^{5}$

T. Cuhadar Donszelmann, ${ }^{149}$ A. R. Cukierman, ${ }^{153}$ S. Czekierda, ${ }^{84}$ P. Czodrowski, ${ }^{36}$ M. J. Da Cunha Sargedas De Sousa, ${ }^{60 b}$ J. V. Da Fonseca Pinto, ${ }^{80 \mathrm{~b}}$ C. Da Via, ${ }^{100}$ W. Dabrowski, ${ }^{83 a}$ T. Dado ${ }^{28 a}$ S. Dahbi, ${ }^{35 \mathrm{e}}$ T. Dai, ${ }^{105}$ C. Dallapiccola,${ }^{102}$ M. Dam, ${ }^{40}$ G. D'amen, ${ }^{23 b, 23 a}$ V. D'Amico, ${ }^{74 a, 74 b}$ J. Damp, ${ }^{99}$ J. R. Dandoy, ${ }^{137}$ M. F. Daneri, ${ }^{30}$ N. P. Dang, ${ }^{181}$ N. D Dann, ${ }^{100}$ M. Danninger ${ }^{175}$ V. Dao, ${ }^{36}$ G. Darbo ${ }^{55 \mathrm{~b}}$ O. Dartsi,${ }^{5}$ A. Dattagupta, ${ }^{131}$ T. Daubney, ${ }^{46}$ S. D'Auria,${ }^{68 a, 68 b}$ W. Davey, ${ }^{24}$ C. David, ${ }^{46}$ T. Davidek, ${ }^{143}$ D. R. Davis,${ }^{49}$ I. Dawson, ${ }^{149}$ K. De,${ }^{8}$ R. De Asmundis, ${ }^{69 a}$ M. De Beurs,${ }^{120}$ S. De Castro, ${ }^{23 b, 23 a}$ S. De Cecco, ${ }^{72 a, 72 b}$ N. De Groot, ${ }^{119}$ P. de Jong, ${ }^{120}$ H. De la Torre, ${ }^{106}$ A. De Maria, ${ }^{15 c}$ D. De Pedis, ${ }^{72 a}$ A. De Salvo, ${ }^{72 a}$ U. De Sanctis ${ }^{73 a, 73 b}$ M. De Santis ${ }^{73 a, 73 b}$ A. De Santo, ${ }^{156}$ K. De Vasconcelos Corga, ${ }^{101}$ J. B. De Vivie De Regie, ${ }^{132}$ C. Debenedetti, ${ }^{146}$ D. V. Dedovich, ${ }^{79}$ A. M. Deiana, ${ }^{42}$ M. Del Gaudio, ${ }^{41 b, 41 a}$ J. Del Peso, ${ }^{98}$ Y. Delabat Diaz, ${ }^{46}$ D. Delgove, ${ }^{132}$ F. Deliot, ${ }^{145, \mathrm{q}}$ C. M. Delitzsch, ${ }^{7}$ M. Della Pietra, ${ }^{69 \mathrm{a}, 69 \mathrm{~b}}$ D. Della Volpe, ${ }^{54}$ A. Dell'Acqua, ${ }^{36}$ L. Dell'Asta, ${ }^{73 a, 73 b}$ M. Delmastro, ${ }^{5}$ C. Delporte, ${ }^{132}$ P. A. Delsart, ${ }^{58}$ D. A. DeMarco, ${ }^{167}$ S. Demers,${ }^{183}$ M. Demichev, ${ }^{79}$ G. Demontigny, ${ }^{109}$ S. P. Denisov, ${ }^{123}$ D. Denysiuk, ${ }^{120}$ L. D’Eramo, ${ }^{136}$ D. Derendarz, ${ }^{84}$ J. E. Derkaoui, ${ }^{35 d}$ F. Derue, ${ }^{136}$ P. Dervan, ${ }^{90}$ K. Desch,${ }^{24}$ C. Deterre, ${ }^{46}$ 
K. Dette, ${ }^{167}$ C. Deutsch,${ }^{24}$ M. R. Devesa, ${ }^{30}$ P. O. Deviveiros, ${ }^{36}$ A. Dewhurst, ${ }^{144}$ S. Dhaliwal, ${ }^{26}$ F. A. Di Bello, ${ }^{54}$ A. Di Ciaccio,${ }^{73 a, 73 b}$ L. Di Ciaccio, ${ }^{5}$ W. K. Di Clemente, ${ }^{137}$ C. Di Donato, ${ }^{69 a, 69 b}$ A. Di Girolamo, ${ }^{36}$ G. Di Gregorio, ${ }^{71 a, 71 b}$ B. Di Micco,${ }^{74 a}{ }^{74 b}$ R. Di Nardo, ${ }^{102}$ K. F. Di Petrillo, ${ }^{59}$ R. Di Sipio, ${ }^{167}$ D. Di Valentino, ${ }^{34}$ C. Diaconu, ${ }^{101}$ F. A. Dias, ${ }^{40}$ T. Dias Do Vale, ${ }^{140 a}$ M. A. Diaz, ${ }^{147 a}$ J. Dickinson, ${ }^{18}$ E. B. Diehl,${ }^{105}$ J. Dietrich, ${ }^{19}$ S. Díez Cornell, ${ }^{46}$ A. Dimitrievska, ${ }^{18}$ W. Ding, ${ }^{15 b}$ J. Dingfelder, ${ }^{24}$ F. Dittus, ${ }^{36}$ F. Djama, ${ }^{101}$ T. Djobava, ${ }^{159 b}$ J. I. Djuvsland, ${ }^{17}$ M. A. B. Do Vale,${ }^{80 c}$ M. Dobre, ${ }^{27 b}$ D. Dodsworth, ${ }^{26}$ C. Doglioni, ${ }^{96}$ J. Dolejsi,${ }^{143}$ Z. Dolezal, ${ }^{143}$ M. Donadelli, ${ }^{80 d}$ J. Donini, ${ }^{38}$ A. D'onofrio, ${ }^{92}$ M. D'Onofrio, ${ }^{90}$ J. Dopke, ${ }^{144}$ A. Doria,${ }^{69 a}$ M. T. Dova, ${ }^{88}$ A. T. Doyle,${ }^{57}$ E. Drechsler, ${ }^{152}$ E. Dreyer, ${ }^{152}$ T. Dreyer, ${ }^{53}$ A. S. Drobac, ${ }^{170}$ Y. Duan,${ }^{60 b}$ F. Dubinin, ${ }^{110}$ M. Dubovsky, ${ }^{28 a}$ A. Dubreuil,${ }^{54}$ E. Duchovni, ${ }^{180}$ G. Duckeck, ${ }^{114}$ A. Ducourthial, ${ }^{136}$ O. A. Ducu, ${ }^{109}$ D. Duda ${ }^{115}$ A. Dudarev ${ }^{36}$ A. C. Dudder, ${ }^{99}$ E. M. Duffield, ${ }^{18}$ L. Duflot,${ }^{132}$ M. Dührssen,${ }^{36}$ C. Dülsen, ${ }^{182}$ M. Dumancic, ${ }^{180}$ A. E. Dumitriu, ${ }^{27 b}$ A. K. Duncan, ${ }^{57}$ M. Dunford, ${ }^{61 \mathrm{a}}$ A. Duperrin, ${ }^{101}$ H. Duran Yildiz, ${ }^{4 \mathrm{a}}$ M. Düren, ${ }^{56}$ A. Durglishvili, ${ }^{159 b}$ D. Duschinger, ${ }^{48}$ B. Dutta, ${ }^{46}$ D. Duvnjak, ${ }^{1}$ G. I. Dyckes, ${ }^{137}$ M. Dyndal, ${ }^{36}$ S. Dysch, ${ }^{100}$ B. S. Dziedzic, ${ }^{84}$ K. M. Ecker, ${ }^{115}$ R. C. Edgar, ${ }^{105}$ T. Eifert, ${ }^{36}$ G. Eigen,${ }^{17}$ K. Einsweiler,${ }^{18}$ T. Ekelof, ${ }^{172}$ M. El Kacimi, ${ }^{35 c}$ R. El Kosseifi, ${ }^{101}$ V. Ellajosyula, ${ }^{172}$ M. Ellert, ${ }^{172}$ F. Ellinghaus, ${ }^{182}$ A. A. Elliot, ${ }^{92}$ N. Ellis, ${ }^{36}$ J. Elmsheuser, ${ }^{29}$ M. Elsing, ${ }^{36}$ D. Emeliyanov, ${ }^{144}$ A. Emerman,${ }^{39}$ Y. Enari, ${ }^{163}$ J. S. Ennis, ${ }^{178}$ M. B. Epland, ${ }^{49}$ J. Erdmann, ${ }^{47}$ A. Ereditato, ${ }^{20}$ M. Errenst, ${ }^{36}$ M. Escalier, ${ }^{132}$ C. Escobar, ${ }^{174}$ O. Estrada Pastor, ${ }^{174}$ E. Etzion, ${ }^{161}$ H. Evans ${ }^{65}$ A. Ezhilov, ${ }^{138}$ F. Fabbri, ${ }^{57}$ L. Fabbri, ${ }^{23 b, 23 a}$ V. Fabiani, ${ }^{119}$ G. Facini, ${ }^{94}$ R. M. Faisca Rodrigues Pereira, ${ }^{140 a}$ R. M. Fakhrutdinov, ${ }^{123}$ S. Falciano ${ }^{72 a}$ P. J. Falke, ${ }^{5}$ S. Falke, ${ }^{5}$ J. Faltova,${ }^{143}$ Y. Fang, ${ }^{15 a}$ Y. Fang, ${ }^{15 a}$ G. Fanourakis, ${ }^{44}$ M. Fanti, ${ }^{68 a, 68 b}$ A. Farbin, ${ }^{8}$ A. Farilla, ${ }^{74 a}$ E. M. Farina,${ }^{70 a, 70 b}$ T. Farooque ${ }^{106}$ S. Farrell, ${ }^{18}$ S. M. Farrington, ${ }^{178}$ P. Farthouat, ${ }^{36}$ F. Fassi, ${ }^{35 e}$ P. Fassnacht,${ }^{36}$ D. Fassouliotis, ${ }^{9}$ M. Faucci Giannelli, ${ }^{50}$ W. J. Fawcett,${ }^{32}$ L. Fayard, ${ }^{132}$ O. L. Fedin, ${ }^{138, \mathrm{r}}$ W. Fedorko, ${ }^{175}$ M. Feickert, ${ }^{42}$ S. Feigl, ${ }^{134}$ L. Feligioni, ${ }^{101}$ A. Fell, ${ }^{149}$ C. Feng, ${ }^{60 b}$ E. J. Feng, ${ }^{36}$ M. Feng, ${ }^{49}$ M. J. Fenton, ${ }^{57}$ A. B. Fenyuk, ${ }^{123}$ J. Ferrando, ${ }^{46}$ A. Ferrante, ${ }^{173}$ A. Ferrari, ${ }^{172}$ P. Ferrari, ${ }^{120}$ R. Ferrari, ${ }^{70 a}$ D. E. Ferreira de Lima, ${ }^{61 b}$ A. Ferrer, ${ }^{174}$ D. Ferrere,${ }^{54}$ C. Ferretti, ${ }^{105}$ F. Fiedler, ${ }^{99}$ A. Filipčič,${ }^{91}$ F. Filthaut, ${ }^{119}$ K. D. Finelli, ${ }^{25}$ M. C. N. Fiolhais, ${ }^{140 a}$ L. Fiorini, ${ }^{174}$ F. Fischer, ${ }^{114}$ W. C. Fisher, ${ }^{106}$ I. Fleck, ${ }^{151}$ P. Fleischmann, ${ }^{105}$ R. R. M. Fletcher, ${ }^{137}$

T. Flick, ${ }^{182}$ B. M. Flierl, ${ }^{114}$ L. F. Flores, ${ }^{137}$ L. R. Flores Castillo, ${ }^{63 a}$ F. M. Follega,${ }^{75 a, 75 b}$ N. Fomin, ${ }^{17}$ J. H. Foo, ${ }^{167}$ G. T. Forcolin, ${ }^{75 a, 75 b}$ A. Formica, ${ }^{145}$ F. A. Förster, ${ }^{14}$ A. C. Forti, ${ }^{100}$ A. G. Foster ${ }^{21}$ M. G. Foti, ${ }^{135}$ D. Fournier, ${ }^{132}$ H. Fox, ${ }^{89}$ P. Francavilla, ${ }^{71 a, 71 b}$ S. Francescato, ${ }^{72 a, 72 b}$ M. Franchini, ${ }^{23 b, 23 a}$ S. Franchino, ${ }^{61 a}$ D. Francis, ${ }^{36}$ L. Franconi,${ }^{20}$ M. Franklin, ${ }^{59}$ A. N. Fray, ${ }^{92}$ B. Freund, ${ }^{109}$ W. S. Freund, ${ }^{80 b}$ E. M. Freundlich, ${ }^{47}$ D. C. Frizzell, ${ }^{128}$ D. Froidevaux, ${ }^{36}$ J. A. Frost, ${ }^{135}$ C. Fukunaga ${ }^{164}$ E. Fullana Torregrosa, ${ }^{174}$ E. Fumagalli, ${ }^{55 b, 55 a}$ T. Fusayasu, ${ }^{116}$ J. Fuster,${ }^{174}$ A. Gabrielli, ${ }^{23 b, 23 a}$ A. Gabrielli, ${ }^{18}$ G. P. Gach, ${ }^{83 a}$ S. Gadatsch,${ }^{54}$ P. Gadow, ${ }^{115}$ G. Gagliardi, ${ }^{55 b, 55 a}$ L. G. Gagnon, ${ }^{109}$ C. Galea, ${ }^{27 b}$ B. Galhardo, ${ }^{140 a}$ G. E. Gallardo, ${ }^{135}$ E. J. Gallas, ${ }^{135}$ B. J. Gallop, ${ }^{144}$ P. Gallus, ${ }^{142}$ G. Galster, ${ }^{40}$ R. Gamboa Goni, ${ }^{92}$ K. K. Gan, ${ }^{126}$ S. Ganguly, ${ }^{180}$ J. Gao, ${ }^{60 a}$ Y. Gao,${ }^{90}$ Y. S. Gao, ${ }^{31, h}$ C. García, ${ }^{174}$ J. E. García Navarro, ${ }^{174}$ J. A. García Pascual, ${ }^{15 a}$ C. Garcia-Argos,${ }^{52}$ M. Garcia-Sciveres, ${ }^{18}$ R. W. Gardner,${ }^{37}$ N. Garelli, ${ }^{153}$ S. Gargiulo, ${ }^{52}$ V. Garonne, ${ }^{134}$ A. Gaudiello, ${ }^{55,55 a}$ G. Gaudio, ${ }^{70 a}$ I. L. Gavrilenko, ${ }^{110}$ A. Gavrilyuk, ${ }^{111}$ C. Gay, ${ }^{175}$ G. Gaycken, ${ }^{24}$ E. N. Gazis, ${ }^{10}$ A. A. Geanta, ${ }^{27 b}$ C. N. P. Gee,${ }^{144}$ J. Geisen,${ }^{53}$ M. Geisen, ${ }^{99}$ M. P. Geisler, ${ }^{61 \mathrm{a}}$ C. Gemme, ${ }^{55 \mathrm{~b}}$ M. H. Genest,${ }^{58}$ C. Geng, ${ }^{105}$ S. Gentile, ${ }^{72 \mathrm{a}, 72 \mathrm{~b}}$ S. George, ${ }^{93}$ T. Geralis, ${ }^{44}$ L. O. Gerlach, ${ }^{53}$ P. Gessinger-Befurt, ${ }^{99}$ G. Gessner, ${ }^{47}$ S. Ghasemi, ${ }^{151}$ M. Ghasemi Bostanabad, ${ }^{176}$ M. Ghneimat, ${ }^{24}$ A. Ghosh, ${ }^{77}$ B. Giacobbe, ${ }^{23 b}$ S. Giagu, ${ }^{72 a, 72 b}$ N. Giangiacomi, ${ }^{23 b, 23 a}$ P. Giannetti ${ }^{71 a}$ A. Giannini, ${ }^{69 a, 69 b}$ S. M. Gibson, ${ }^{93}$ M. Gignac, ${ }^{146}$ D. Gillberg, ${ }^{34}$ G. Gilles, ${ }^{182}$ D. M. Gingrich, ${ }^{3, e}$ M. P. Giordani, ${ }^{66 a, 66 c}$ F. M. Giorgi, ${ }^{23 b}$ P. F. Giraud, ${ }^{145}$ G. Giugliarelli, ${ }^{66 a, 66 c}$ D. Giugni, ${ }^{68 a}$ F. Giuli, ${ }^{73 a, 73 b}$ S. Gkaitatzis, ${ }^{162}$ I. Gkialas, ${ }^{9, s}$ E. L. Gkougkousis, ${ }^{14}$ P. Gkountoumis, ${ }^{10}$ L. K. Gladilin, ${ }^{113}$ C. Glasman, ${ }^{98}$ J. Glatzer, ${ }^{14}$ P. C. F. Glaysher, ${ }^{46}$ A. Glazov,${ }^{46}$ M. Goblirsch-Kolb, ${ }^{26}$ S. Goldfarb, ${ }^{104}$ T. Golling, ${ }^{54}$ D. Golubkov, ${ }^{123}$ A. Gomes, ${ }^{140 a, 140 b}$ R. Goncalves Gama, ${ }^{53}$ R. Gonçalo, ${ }^{140 a, 140 b}$ G. Gonella, ${ }^{52}$ L. Gonella, ${ }^{21}$ A. Gongadze ${ }^{79}$ F. Gonnella, ${ }^{21}$ J. L. Gonski, ${ }^{59}$ S. González de la Hoz, ${ }^{174}$ S. Gonzalez-Sevilla, ${ }^{54}$ G. R. Gonzalvo Rodriguez, ${ }^{174}$ L. Goossens,${ }^{36}$ P. A. Gorbounov, ${ }^{111}$ H. A. Gordon, ${ }^{29}$ B. Gorini, ${ }^{36}$ E. Gorini, ${ }^{67 a, 67 b}$ A. Gorišek, ${ }^{91}$ A. T. Goshaw, ${ }^{49}$ C. Gössling, ${ }^{47}$ M. I. Gostkin, ${ }^{79}$ C. A. Gottardo, ${ }^{24}$ M. Gouighri, ${ }^{35 b}$ D. Goujdami, ${ }^{35 c}$ A. G. Goussiou, ${ }^{148}$ N. Govender, ${ }^{33 b, t}$ C. Goy, ${ }^{5}$ E. Gozani, ${ }^{160}$ I. Grabowska-Bold, ${ }^{83 a}$ E. C. Graham, ${ }^{90}$ J. Gramling, ${ }^{171}$ E. Gramstad, ${ }^{134}$ S. Grancagnolo, ${ }^{19}$ M. Grandi, ${ }^{156}$ V. Gratchev, ${ }^{138}$ P. M. Gravila, ${ }^{27 f}$ F. G. Gravili, ${ }^{67 a, 67 b}$ C. Gray, ${ }^{57}$ H. M. Gray, ${ }^{18}$ C. Grefe, ${ }^{24}$ K. Gregersen, ${ }^{96}$ I. M. Gregor,${ }^{46}$ P. Grenier,${ }^{153}$ K. Grevtsov, ${ }^{46}$ C. Grieco, ${ }^{14}$ N. A. Grieser, ${ }^{128}$ J. Griffiths, ${ }^{8}$ A. A. Grillo, ${ }^{146}$ K. Grimm, ${ }^{31, u}$ S. Grinstein, ${ }^{14, v}$ J.-F. Grivaz, ${ }^{132}$ S. Groh, ${ }^{99}$ E. Gross, ${ }^{180}$ J. Grosse-Knetter, ${ }^{53}$ Z. J. Grout, ${ }^{94}$ C. Grud,${ }^{105}$ A. Grummer, ${ }^{118}$ L. Guan, ${ }^{105}$ W. Guan, ${ }^{181}$ J. Guenther, ${ }^{36}$ A. Guerguichon, ${ }^{132}$ F. Guescini, ${ }^{115}$ D. Guest, ${ }^{171}$ R. Gugel, ${ }^{52}$ T. Guillemin, ${ }^{5}$ S. Guindon, ${ }^{36}$ U. Gul, ${ }^{57}$ J. Guo, ${ }^{60 \mathrm{c}}$ W. Guo, ${ }^{105}$ Y. Guo, ${ }^{60 a, w}$ Z. Guo, ${ }^{101}$ R. Gupta, ${ }^{46}$ S. Gurbuz, ${ }^{12 c}$ G. Gustavino, ${ }^{128}$ P. Gutierrez, ${ }^{128}$ C. Gutschow, ${ }^{94}$ C. Guyot, ${ }^{145}$ M. P. Guzik, ${ }^{83 a}$ C. Gwenlan, ${ }^{135}$ 
C. B. Gwilliam, ${ }^{90}$ A. Haas, ${ }^{124}$ C. Haber, ${ }^{18}$ H. K. Hadavand, ${ }^{8}$ N. Haddad, ${ }^{35 e}$ A. Hadef ${ }^{60 a}$ S. Hageböck,${ }^{36}$ M. Hagihara, ${ }^{169}$ M. Haleem, ${ }^{177}$ J. Haley, ${ }^{129}$ G. Halladjian, ${ }^{106}$ G. D. Hallewell, ${ }^{101}$ K. Hamacher, ${ }^{182}$ P. Hamal, ${ }^{130}$ K. Hamano, ${ }^{176}$ H. Hamdaoui, ${ }^{35 \mathrm{e}}$ G. N. Hamity, ${ }^{149}$ K. Han,${ }^{60 \mathrm{a}, \mathrm{x}}$ L. Han, ${ }^{60 \mathrm{a}}$ S. Han, ${ }^{15 a, 15 \mathrm{~d}}$ K. Hanagaki, ${ }^{81, y}$ M. Hance, ${ }^{146}$ D. M. Handl, ${ }^{114}$ B. Haney, ${ }^{137}$ R. Hankache, ${ }^{136}$ P. Hanke, ${ }^{61 a}$ E. Hansen, ${ }^{96}$ J. B. Hansen, ${ }^{40}$ J. D. Hansen, ${ }^{40}$ M. C. Hansen, ${ }^{24}$ P. H. Hansen, ${ }^{40}$ E. C. Hanson, ${ }^{100}$ K. Hara, ${ }^{169}$ A. S. Hard ${ }^{181}$ T. Harenberg, ${ }^{182}$ S. Harkusha, ${ }^{107}$ P. F. Harrison, ${ }^{178}$ N. M. Hartmann, ${ }^{114}$ Y. Hasegawa, ${ }^{150}$ A. Hasib ${ }^{50}$ S. Hassani, ${ }^{145}$ S. Haug, ${ }^{20}$ R. Hauser, ${ }^{106}$ L. B. Havener, ${ }^{39}$ M. Havranek, ${ }^{142}$ C. M. Hawkes,${ }^{21}$ R. J. Hawkings, ${ }^{36}$ D. Hayden, ${ }^{106}$ C. Hayes,${ }^{155}$ R. L. Hayes, ${ }^{175}$ C. P. Hays, ${ }^{135}$ J. M. Hays,${ }^{92}$ H. S. Hayward, ${ }^{90}$ S. J. Haywood, ${ }^{144}$ F. He, ${ }^{60 a}$ M. P. Heath, ${ }^{50}$ V. Hedberg, ${ }^{96}$ L. Heelan, ${ }^{8}$ S. Heer,${ }^{24}$ K. K. Heidegger, ${ }^{52}$ W. D. Heidorn, ${ }^{78}$ J. Heilman, ${ }^{34}$ S. Heim, ${ }^{46}$ T. Heim, ${ }^{18}$ B. Heinemann, ${ }^{46, z}$ J. J. Heinrich,${ }^{131}$ L. Heinrich, ${ }^{36}$ C. Heinz, ${ }^{56}$ J. Hejbal, ${ }^{141}$ L. Helary, ${ }^{61 b}$ A. Held, ${ }^{175}$ S. Hellesund, ${ }^{134}$ C. M. Helling, ${ }^{146}$ S. Hellman, ${ }^{45 a, 45 b}$ C. Helsens, ${ }^{36}$ R. C. W. Henderson, ${ }^{89}$ Y. Heng, ${ }^{181}$ S. Henkelmann, ${ }^{175}$ A. M. Henriques Correia, ${ }^{36}$ G. H. Herbert, ${ }^{19}$ H. Herde, ${ }^{26}$ V. Herget, ${ }^{177}$ Y. Hernández Jiménez, ${ }^{33 c}$

H. Herr, ${ }^{99}$ M. G. Herrmann, ${ }^{114}$ T. Herrmann, ${ }^{48}$ G. Herten, ${ }^{52}$ R. Hertenberger, ${ }^{114}$ L. Hervas, ${ }^{36}$ T. C. Herwig, ${ }^{137}$ G. G. Hesketh, ${ }^{94}$ N. P. Hessey, ${ }^{168 a}$ A. Higashida, ${ }^{163}$ S. Higashino, ${ }^{81}$ E. Higón-Rodriguez, ${ }^{174}$ K. Hildebrand, ${ }^{37}$ E. Hill, ${ }^{176}$ J. C. Hill, ${ }^{32}$ K. K. Hill, ${ }^{29}$ K. H. Hiller, ${ }^{46}$ S. J. Hillier,${ }^{21}$ M. Hils,${ }^{48}$ I. Hinchliffe, ${ }^{18}$ F. Hinterkeuser, ${ }^{24}$ M. Hirose, ${ }^{133}$ S. Hirose,${ }^{52}$ D. Hirschbuehl, ${ }^{182}$ B. Hiti, ${ }^{91}$ O. Hladik, ${ }^{141}$ D. R. Hlaluku, ${ }^{33 c}$ X. Hoad,${ }^{50}$ J. Hobbs,${ }^{155}$ N. Hod, ${ }^{180}$ M. C. Hodgkinson, ${ }^{149}$ A. Hoecker, ${ }^{36}$ F. Hoenig, ${ }^{114}$ D. Hohn, ${ }^{52}$ D. Hohov, ${ }^{132}$ T. R. Holmes, ${ }^{37}$ M. Holzbock, ${ }^{114}$ L. B. A. H Hommels,${ }^{32}$ S. Honda, ${ }^{169}$ T. Honda ${ }^{81}$ T. M. Hong, ${ }^{139}$ A. Hönle, ${ }^{115}$ B. H. Hooberman, ${ }^{173}$ W. H. Hopkins, ${ }^{6}$ Y. Horii, ${ }^{117}$ P. Horn, ${ }^{48}$ L. A. Horyn,${ }^{37}$ J-Y. Hostachy, ${ }^{58}$ A. Hostiuc, ${ }^{148}$ S. Hou, ${ }^{158}$ A. Hoummada, ${ }^{35 a}$ J. Howarth, ${ }^{100}$ J. Hoya ${ }^{88}$ M. Hrabovsky, ${ }^{130}$ J. Hrdinka, ${ }^{76}$ I. Hristova, ${ }^{19}$ J. Hrivnac, ${ }^{132}$ A. Hrynevich, ${ }^{108}$ T. Hryn'ova, ${ }^{5}$ P. J. Hsu, ${ }^{64}$ S.-C. Hsu, ${ }^{148}$ Q. Hu, ${ }^{29}$ S. Hu, ${ }^{60 c}$ Y. Huang, ${ }^{15 a}$ Z. Hubacek, ${ }^{142}$ F. Hubaut, ${ }^{101}$ M. Huebner, ${ }^{24}$ F. Huegging, ${ }^{24}$ T. B. Huffman ${ }^{135}$ M. Huhtinen, ${ }^{36}$ R. F. H. Hunter,${ }^{34}$ P. Huo, ${ }^{155}$ A. M. Hupe, ${ }^{34}$ N. Huseynov, ${ }^{79, a a}$ J. Huston, ${ }^{106}$ J. Huth,${ }^{59}$ R. Hyneman, ${ }^{105}$ S. Hyrych ${ }^{28 a}$ G. Iacobucci,${ }^{54}$ G. Iakovidis, ${ }^{29}$ I. Ibragimov, ${ }^{151}$ L. Iconomidou-Fayard, ${ }^{132}$ Z. Idrissi, ${ }^{35 e}$ P. I. Iengo, ${ }^{36}$ R. Ignazzi, ${ }^{40}$ O. Igonkina, ${ }^{120, b b}$ R. Iguchi, ${ }^{163}$ T. Iizawa, ${ }^{54}$ Y. Ikegami,${ }^{81}$ M. Ikeno, ${ }^{81}$ D. Iliadis, ${ }^{162} \mathrm{~N}$. Ilic, ${ }^{119}$ F. Iltzsche, ${ }^{48}$ G. Introzzi, ${ }^{70 a, 70 b}$ M. Iodice,${ }^{74 a} \mathrm{~K}$. Iordanidou, ${ }^{168 \mathrm{a}}$ V. Ippolito, ${ }^{72 a, 72 b}$ M. F. Isacson, ${ }^{172} \mathrm{M}$. Ishino, ${ }^{163} \mathrm{M}$. Ishitsuka, ${ }^{165} \mathrm{~W}$. Islam, ${ }^{129} \mathrm{C}$. Issever, ${ }^{135} \mathrm{~S}$. Istin, ${ }^{160} \mathrm{~F}$. Ito, ${ }^{169}$ J. M. Iturbe Ponce, ${ }^{63 a}$ R. Iuppa, ${ }^{75 a, 75 b}$ A. Ivina, ${ }^{180}$ H. Iwasaki, ${ }^{81}$ J. M. Izen, ${ }^{43}$ V. Izzo, ${ }^{69 a}$ P. Jacka, ${ }^{141}$ P. Jackson, ${ }^{1}$ R. M. Jacobs ${ }^{24}$ B. P. Jaeger, ${ }^{152}$ V. Jain, ${ }^{2}$ G. Jäkel, ${ }^{182}$ K. B. Jakobi, ${ }^{99}$ K. Jakobs, ${ }^{52}$ S. Jakobsen, ${ }^{76}$ T. Jakoubek, ${ }^{141}$ J. Jamieson, ${ }^{57}$ K. W. Janas, ${ }^{83 a}$ R. Jansky, ${ }^{54}$ J. Janssen, ${ }^{24}$ M. Janus, ${ }^{53}$ P. A. Janus, ${ }^{83 a}$ G. Jarlskog, ${ }^{96}$ N. Javadov, ${ }^{79, a a}$ T. Javůrek, ${ }^{36}$ M. Javurkova, ${ }^{52}$ F. Jeanneau, ${ }^{145}$ L. Jeanty, ${ }^{131}$ J. Jejelava, ${ }^{159 a, c c}$ A. Jelinskas, ${ }^{178}$ P. Jenni, ${ }^{52, d d}$ J. Jeong, ${ }^{46}$ N. Jeong, ${ }^{46}$ S. Jézéquel,,${ }^{5}$ H. Ji, ${ }^{181}$ J. Jia ${ }^{155}$ H. Jiang, ${ }^{78}$ Y. Jiang, ${ }^{60 a}$ Z. Jiang, ${ }^{153, \text { ee }}$ S. Jiggins, ${ }^{52}$ F. A. Jimenez Morales, ${ }^{38}$ J. Jimenez Pena, ${ }^{174}$ S. Jin, ${ }^{15 c}$ A. Jinaru, ${ }^{27 b}$ O. Jinnouchi, ${ }^{165}$ H. Jivan, ${ }^{33 c}$ P. Johansson, ${ }^{149}$ K. A. Johns, ${ }^{7}$ C. A. Johnson, ${ }^{65}$ K. Jon-And, ${ }^{45 a, 45 b}$ R. W. L. Jones, ${ }^{89}$ S. D. Jones, ${ }^{156}$ S. Jones, ${ }^{7}$ T. J. Jones, ${ }^{90}$ J. Jongmanns, ${ }^{61 a}$ P. M. Jorge, ${ }^{140 a}$ J. Jovicevic, ${ }^{36}$ X. Ju, ${ }^{18}$ J. J. Junggeburth, ${ }^{115}$ A. Juste Rozas, ${ }^{14, v}$ A. Kaczmarska, ${ }^{84}$ M. Kado, ${ }^{72 a, 72 b}$ H. Kagan, ${ }^{126}$ M. Kagan, ${ }^{153}$ C. Kahra, ${ }^{99}$

T. Kaji, ${ }^{179}$ E. Kajomovitz, ${ }^{160}$ C. W. Kalderon, ${ }^{96}$ A. Kaluza, ${ }^{99}$ A. Kamenshchikov, ${ }^{123}$ L. Kanjir,${ }^{91}$ Y. Kano, ${ }^{163}$

V. A. Kantserov, ${ }^{112}$ J. Kanzaki, ${ }^{81}$ L. S. Kaplan, ${ }^{181}$ D. Kar, ${ }^{33 \mathrm{c}}$ M. J. Kareem, ${ }^{168 \mathrm{~b}}$ E. Karentzos, ${ }^{10}$ S. N. Karpov, ${ }^{79}$ Z. M. Karpova, ${ }^{79}$ V. Kartvelishvili, ${ }^{89}$ A. N. Karyukhin, ${ }^{123}$ L. Kashif, ${ }^{181}$ R. D. Kass, ${ }^{126}$ A. Kastanas, ${ }^{45 a, 45 b}$ Y. Kataoka, ${ }^{163}$ C. Kato, ${ }^{60 d, 60 c}$ J. Katzy, ${ }^{46}$ K. Kawade, ${ }^{82}$ K. Kawagoe, ${ }^{87}$ T. Kawaguchi, ${ }^{117}$ T. Kawamoto, ${ }^{163}$ G. Kawamura ${ }^{53}$ E. F. Kay, ${ }^{176}$ V. F. Kazanin, ${ }^{122 b, 122 a}$ R. Keeler, ${ }^{176}$ R. Kehoe, ${ }^{42}$ J. S. Keller, ${ }^{34}$ E. Kellermann, ${ }^{96}$ D. Kelsey, ${ }^{156}$ J. J. Kempster, ${ }^{21}$ J. Kendrick, ${ }^{21}$ O. Kepka, ${ }^{141}$ S. Kersten, ${ }^{182}$ B. P. Kerševan, ${ }^{91}$ S. Ketabchi Haghighat, ${ }^{167}$ M. Khader ${ }^{173}$ F. Khalil-Zada, ${ }^{13}$ M. K. Khandoga, ${ }^{145}$ A. Khanov, ${ }^{129}$ A. G. Kharlamov, ${ }^{122 b, 122 a}$ T. Kharlamova, ${ }^{122 b, 122 \mathrm{a}}$ E. E. Khoda, ${ }^{175}$ A. Khodinov, ${ }^{166}$ T. J. Khoo, ${ }^{54}$ E. Khramov, ${ }^{79}$ J. Khubua, ${ }^{159 b}$ S. Kido,${ }^{82}$ M. Kiehn, ${ }^{54}$ C. R. Kilby,${ }^{93}$ Y. K. Kim, ${ }^{37}$ N. Kimura, ${ }^{66 a, 66 c}$ O. M. Kind, ${ }^{19}$ B. T. King, ${ }^{90, a}$

D. Kirchmeier, ${ }^{48}$ J. Kirk, ${ }^{144}$ A. E. Kiryunin, ${ }^{115}$ T. Kishimoto, ${ }^{163}$ D. P. Kisliuk,${ }^{167}$ V. Kitali, ${ }^{46}$ O. Kivernyk, ${ }^{5}$ E. Kladiva, ${ }^{28 b, a}$ T. Klapdor-Kleingrothaus, ${ }^{52}$ M. Klassen, ${ }^{61 a}$ M. H. Klein, ${ }^{105}$ M. Klein, ${ }^{90}$ U. Klein, ${ }^{90}$ K. Kleinknecht, ${ }^{99}$ P. Klimek, ${ }^{121}$

A. Klimentov, ${ }^{29}$ T. Klingl, ${ }^{24}$ T. Klioutchnikova, ${ }^{36}$ F. F. Klitzner, ${ }^{114}$ P. Kluit, ${ }^{120}$ S. Kluth, ${ }^{115}$ E. Kneringer,${ }^{76}$

E. B. F. G. Knoops, ${ }^{101}$ A. Knue,${ }^{52}$ D. Kobayashi, ${ }^{87}$ T. Kobayashi, ${ }^{163}$ M. Kobel,${ }^{48}$ M. Kocian, ${ }^{153}$ P. Kodys ${ }^{143}$ P. T. Koenig, ${ }^{24}$ T. Koffas,${ }^{34}$ N. M. Köhler, ${ }^{115}$ T. Koi, ${ }^{153}$ M. Kolb,${ }^{61 b}$ I. Koletsou, ${ }^{5}$ T. Komarek, ${ }^{130}$ T. Kondo, ${ }^{81}$ N. Kondrashova, ${ }^{60 \mathrm{c}}$ K. Köneke, ${ }^{52}$ A. C. König, ${ }^{119}$ T. Kono, ${ }^{125}$ R. Konoplich, ${ }^{124, f f}$ V. Konstantinides,${ }^{94}$ N. Konstantinidis, ${ }^{94}$ B. Konya, ${ }^{96}$ R. Kopeliansky, ${ }^{65}$ S. Koperny, ${ }^{83 a}$ K. Korcyl ${ }^{84}$ K. Kordas, ${ }^{162}$ G. Koren, ${ }^{161}$ A. Korn, ${ }^{94}$ I. Korolkov, ${ }^{14}$ E. V. Korolkova, ${ }^{149}$ N. Korotkova, ${ }^{113}$ O. Kortner, ${ }^{115}$ S. Kortner, ${ }^{115}$ T. Kosek, ${ }^{143}$ V. V. Kostyukhin,${ }^{24}$ A. Kotwal, ${ }^{49}$ A. Koulouris, ${ }^{10}$

A. Kourkoumeli-Charalampidi, ${ }^{70 a, 70 b}$ C. Kourkoumelis, ${ }^{9}$ E. Kourlitis, ${ }^{149}$ V. Kouskoura, ${ }^{29}$ A. B. Kowalewska, ${ }^{84}$ 
R. Kowalewski, ${ }^{176}$ C. Kozakai, ${ }^{163}$ W. Kozanecki, ${ }^{145}$ A. S. Kozhin, ${ }^{123}$ V. A. Kramarenko, ${ }^{113}$ G. Kramberger, ${ }^{91}$ D. Krasnopevtsev, ${ }^{60 a}$ M. W. Krasny, ${ }^{136}$ A. Krasznahorkay, ${ }^{36}$ D. Krauss, ${ }^{115}$ J. A. Kremer, ${ }^{83 a}$ J. Kretzschmar, ${ }^{90}$ P. Krieger, ${ }^{167}$ F. Krieter, ${ }^{114}$ A. Krishnan, ${ }^{61 \mathrm{~b}}$ K. Krizka, ${ }^{18}$ K. Kroeninger, ${ }^{47}$ H. Kroha, ${ }^{115}$ J. Kroll, ${ }^{141}$ J. Kroll, ${ }^{137}$ J. Krstic, ${ }^{16}$ U. Kruchonak, ${ }^{79}$ H. Krüger, ${ }^{24}$ N. Krumnack, ${ }^{78}$ M. C. Kruse, ${ }^{49}$ J. A. Krzysiak, ${ }^{84}$ T. Kubota, ${ }^{104}$ S. Kuday, ${ }^{4 b}$ J. T. Kuechler, ${ }^{46}$ S. Kuehn, ${ }^{36}$ A. Kugel, ${ }^{61 a}$ T. Kuhl,,${ }^{46}$ V. Kukhtin, ${ }^{79}$ R. Kukla, ${ }^{101}$ Y. Kulchitsky, ${ }^{107, g g}$ S. Kuleshov, ${ }^{14 b}$ Y. P. Kulinich, ${ }^{173}$ M. Kuna, ${ }^{58}$

T. Kunigo, ${ }^{85}$ A. Kupco, ${ }^{141}$ T. Kupfer,${ }^{47}$ O. Kuprash, ${ }^{52}$ H. Kurashige, ${ }^{82}$ L. L. Kurchaninov, ${ }^{168 a}$ Y. A. Kurochkin, ${ }^{107}$ A. Kurova, ${ }^{112}$ M. G. Kurth, ${ }^{15 a, 15 d}$ E. S. Kuwertz, ${ }^{36}$ M. Kuze, ${ }^{165}$ A. K. Kvam, ${ }^{148}$ J. Kvita, ${ }^{130}$ T. Kwan, ${ }^{103}$ A. La Rosa, ${ }^{115}$ L. La Rotonda, ${ }^{41 b, 41 a}$ F. La Ruffa, ${ }^{41 b, 41 a}$ C. Lacasta, ${ }^{174}$ F. Lacava, ${ }^{72 a, 72 b}$ D. P. J. Lack, ${ }^{100}$ H. Lacker, ${ }^{19}$ D. Lacour, ${ }^{136}$ E. Ladygin,${ }^{79}$ R. Lafaye, ${ }^{5}$ B. Laforge,${ }^{136}$ T. Lagouri, ${ }^{33 c}$ S. Lai, ${ }^{53}$ S. Lammers, ${ }^{65}$ W. Lampl, ${ }^{7}$ C. Lampoudis,${ }^{162}$ E. Lançon, ${ }^{29}$ U. Landgraf, ${ }^{52}$ M. P. J. Landon, ${ }^{92}$ M. C. Lanfermann, ${ }^{54}$ V. S. Lang, ${ }^{46}$ J. C. Lange, ${ }^{53}$ R. J. Langenberg, ${ }^{36}$ A. J. Lankford, ${ }^{171}$ F. Lanni, ${ }^{29}$ K. Lantzsch, ${ }^{24}$ A. Lanza, ${ }^{70 a}$ A. Lapertosa, ${ }^{55 b, 55 a}$ S. Laplace, ${ }^{136}$ J. F. Laporte ${ }^{145}$ T. Lari, ${ }^{68 a}$ F. Lasagni Manghi, ${ }^{23 b, 23 a}$ M. Lassnig, ${ }^{36}$ T. S. Lau, ${ }^{63 a}$ A. Laudrain, ${ }^{132}$ A. Laurier, ${ }^{34}$ M. Lavorgna ${ }^{69 a, 69 b}$ M. Lazzaroni, ${ }^{68 a, 68 b}$ B. Le ${ }^{104}$ O. Le Dortz, ${ }^{136}$ E. Le Guirriec, ${ }^{101}$ M. LeBlanc, ${ }^{7}$ T. LeCompte, ${ }^{6}$ F. Ledroit-Guillon, ${ }^{58}$ C. A. Lee, ${ }^{29}$ G. R. Lee, ${ }^{17}$

L. Lee, ${ }^{59}$ S. C. Lee, ${ }^{158}$ S. J. Lee, ${ }^{34}$ B. Lefebvre, ${ }^{168 a}$ M. Lefebvre ${ }^{176}$ F. Legger, ${ }^{114}$ C. Leggett, ${ }^{18}$ K. Lehmann, ${ }^{152}$ N. Lehmann, ${ }^{182}$ G. Lehmann Miotto, ${ }^{36}$ W. A. Leight, ${ }^{46}$ A. Leisos, ${ }^{162, \text { hh }}$ M. A. L. Leite, ${ }^{80 d}$ C. E. Leitgeb,${ }^{114}$ R. Leitner, ${ }^{143}$ D. Lellouch, ${ }^{180, a}$ K. J. C. Leney, ${ }^{42}$ T. Lenz,${ }^{24}$ B. Lenzi, ${ }^{36}$ R. Leone, ${ }^{7}$ S. Leone, ${ }^{71 a}$ C. Leonidopoulos, ${ }^{50}$ A. Leopold, ${ }^{136}$

G. Lerner, ${ }^{156}$ C. Leroy, ${ }^{109}$ R. Les, ${ }^{167}$ C. G. Lester,${ }^{32}$ M. Levchenko, ${ }^{138}$ J. Levêque, ${ }^{5}$ D. Levin, ${ }^{105}$ L. J. Levinson, ${ }^{180}$ D. J. Lewis, ${ }^{21}$ B. Li ${ }^{15 b}$ B. Li, ${ }^{105} \mathrm{C}-\mathrm{Q} . \mathrm{Li},{ }^{60 \mathrm{a}} \mathrm{F}$. Li ${ }^{60 \mathrm{c}} \mathrm{H} . \mathrm{Li},{ }^{60 \mathrm{a}} \mathrm{H} . \mathrm{Li},{ }^{60 \mathrm{~b}} \mathrm{~J} . \mathrm{Li},{ }^{60 \mathrm{c}} \mathrm{K} . \mathrm{Li},{ }^{153} \mathrm{~L} . \mathrm{Li},{ }^{60 \mathrm{c}} \mathrm{M} . \mathrm{Li},{ }^{15 \mathrm{a}} \mathrm{Q} . \mathrm{Li},{ }^{15 a}, 15 \mathrm{~d}$ Q. Y. Li, ${ }^{60 \mathrm{a}} \mathrm{S} . \mathrm{Li},{ }^{60 \mathrm{~d}, 60 \mathrm{c}} \mathrm{X} . \mathrm{Li},{ }^{46} \mathrm{Y}_{\mathrm{Li}},{ }^{46} \mathrm{Z}$. Li, ${ }^{60 \mathrm{~b}} \mathrm{Z}$. Liang, ${ }^{15 \mathrm{a}}$ B. Liberti, ${ }^{73 \mathrm{a}}$ A. Liblong,${ }^{167} \mathrm{~K}$. Lie, ${ }^{63 \mathrm{c}} \mathrm{S} . \mathrm{Liem},{ }^{120} \mathrm{C}$. Y. Lin, ${ }^{32}$ K. Lin, ${ }^{106}$ T. H. Lin, ${ }^{99}$ R. A. Linck, ${ }^{65}$ J. H. Lindon, ${ }^{21}$ A. L. Lionti, ${ }^{54}$ E. Lipeles, ${ }^{137}$ A. Lipniacka, ${ }^{17}$ M. Lisovyi, ${ }^{61 b}$ T. M. Liss, ${ }^{173, i i}$ A. Lister ${ }^{175}$ A. M. Litke, ${ }^{146}$ J. D. Little, ${ }^{8}$ B. Liu, ${ }^{78, j j}$ B. L Liu, ${ }^{6}$ H. B. Liu, ${ }^{29}$ H. Liu, ${ }^{105}$ J. B. Liu, ${ }^{60 a}$ J. K. K. Liu, ${ }^{135}$ K. Liu, ${ }^{136}$ M. Liu, ${ }^{60 a}$ P. Liu, ${ }^{18}$ Y. Liu, ${ }^{15 a, 15 d}$ Y. L. Liu, ${ }^{105}$ Y. W. Liu, ${ }^{60 a}$ M. Livan, ${ }^{70 a, 70 b}$ A. Lleres, ${ }^{58}$ J. Llorente Merino, ${ }^{15 a}$ S. L. Lloyd, ${ }^{92}$ C. Y. Lo, ${ }^{63 b}$ F. Lo Sterzo, ${ }^{42}$ E. M. Lobodzinska, ${ }^{46}$ P. Loch, ${ }^{7}$ S. Loffredo, ${ }^{73 a, 73 b}$ T. Lohse, ${ }^{19}$ K. Lohwasser, ${ }^{149}$ M. Lokajicek, ${ }^{141}$ J. D. Long, ${ }^{173}$ R. E. Long, ${ }^{89}$ L. Longo, ${ }^{36}$ K. A. Looper, ${ }^{126}$ J. A. Lopez, ${ }^{147 b}$ I. Lopez Paz, ${ }^{100}$ A. Lopez Solis, ${ }^{149}$ J. Lorenz,${ }^{114}$ N. Lorenzo Martinez, ${ }^{5}$ M. Losada, ${ }^{22}$ P. J. Lösel, ${ }^{114}$ A. Lösle,${ }^{52}$ X. Lou ${ }^{46}$ X. Lou, ${ }^{15 a}$ A. Lounis, ${ }^{132}$ J. Love, ${ }^{6}$ P. A. Love, ${ }^{89}$ J. J. Lozano Bahilo, ${ }^{174}$ M. Lu, ${ }^{60 a}$ Y. J. Lu, ${ }^{64}$ H. J. Lubatti, ${ }^{148}$ C. Luci, ${ }^{72 a, 72 b}$ A. Lucotte,${ }^{58}$ C. Luedtke, ${ }^{52}$ F. Luehring, ${ }^{65}$ I. Luise, ${ }^{136}$ L. Luminari, ${ }^{72 a}$ B. Lund-Jensen, ${ }^{154}$ M. S. Lutz, ${ }^{102}$ D. Lynn, ${ }^{29}$ R. Lysak, ${ }^{141}$ E. Lytken,${ }^{96}$ F. Lyu ${ }^{15 a}$ V. Lyubushkin, ${ }^{79}$ T. Lyubushkina, ${ }^{79}$ H. Ma ${ }^{29}$ L. L. Ma,${ }^{60 b}$ Y. Ma ${ }^{60 b}$ G. Maccarrone,${ }^{51}$ A. Macchiolo, ${ }^{115}$ C. M. Macdonald, ${ }^{149}$ J. Machado Miguens, ${ }^{137}$ D. Madaffari, ${ }^{174}$ R. Madar, ${ }^{38}$ W. F. Mader,${ }^{48}$ N. Madysa ${ }^{48}$ J. Maeda, ${ }^{82}$ K. Maekawa, ${ }^{163}$ S. Maeland,${ }^{17}$ T. Maeno, ${ }^{29}$ M. Maerker, ${ }^{48}$ A. S. Maevskiy, ${ }^{113}$ V. Magerl, ${ }^{52}$ N. Magini ${ }^{78}$ D. J. Mahon, ${ }^{39}$ C. Maidantchik, ${ }^{80 b}$ T. Maier, ${ }^{114}$ A. Maio, ${ }^{140 a, 140 b, 140 d}$ O. Majersky, ${ }^{28 a}$ S. Majewski, ${ }^{131}$ Y. Makida, ${ }^{81}$ N. Makovec, ${ }^{132}$ B. Malaescu, ${ }^{136}$ Pa. Malecki, ${ }^{84}$ V. P. Maleev, ${ }^{138}$ F. Malek, ${ }^{58}$ U. Mallik, ${ }^{77}$ D. Malon, ${ }^{6}$ C. Malone, ${ }^{32}$ S. Maltezos, ${ }^{10}$ S. Malyukov, ${ }^{36}$ J. Mamuzic, ${ }^{174}$ G. Mancini,${ }^{51}$ I. Mandić, ${ }^{91}$ L. Manhaes de Andrade Filho, ${ }^{80 a}$ I. M. Maniatis, ${ }^{162}$ J. Manjarres Ramos, ${ }^{48}$ K. H. Mankinen, ${ }^{96}$ A. Mann, ${ }^{114}$ A. Manousos, ${ }^{76}$ B. Mansoulie, ${ }^{145}$ I. Manthos, ${ }^{162}$ S. Manzoni, ${ }^{120}$ A. Marantis, ${ }^{162}$ G. Marceca, ${ }^{30}$ L. Marchese, ${ }^{135}$ G. Marchiori, ${ }^{136}$ M. Marcisovsky, ${ }^{141}$ C. Marcon, ${ }^{96}$ C. A. Marin Tobon, ${ }^{36}$

M. Marjanovic, ${ }^{38}$ F. Marroquim, ${ }^{80 \mathrm{~b}}$ Z. Marshall, ${ }^{18}$ M. U. F Martensson, ${ }^{172}$ S. Marti-Garcia, ${ }^{174}$ C. B. Martin, ${ }^{126}$

T. A. Martin, ${ }^{178}$ V. J. Martin, ${ }^{50}$ B. Martin dit Latour, ${ }^{17}$ L. Martinelli, ${ }^{74 a, 74 b}$ M. Martinez, ${ }^{14, v}$ V. I. Martinez Outschoorn, ${ }^{102}$ S. Martin-Haugh, ${ }^{144}$ V. S. Martoiu, ${ }^{27 b}$ A. C. Martyniuk, ${ }^{94}$ A. Marzin, ${ }^{36}$ S. R. Maschek, ${ }^{115}$ L. Masetti, ${ }^{99}$ T. Mashimo, ${ }^{163}$

R. Mashinistov, ${ }^{110}$ J. Masik, ${ }^{100}$ A. L. Maslennikov, ${ }^{122 b, 122 a}$ L. H. Mason, ${ }^{104}$ L. Massa, ${ }^{73 a, 73 b}$ P. Massarotti, ${ }^{69,69 b}$ P. Mastrandrea, ${ }^{71 \mathrm{a}, 71 \mathrm{~b}}$ A. Mastroberardino, ${ }^{41 \mathrm{~b}, 41 \mathrm{a}}$ T. Masubuchi, ${ }^{163}$ A. Matic, ${ }^{114}$ P. Mättig, ${ }^{24}$ J. Maurer, ${ }^{27 b}$ B. Maček, ${ }^{91}$ S. J. Maxfield, ${ }^{90}$ D. A. Maximov, ${ }^{122 b, 122 a}$ R. Mazini, ${ }^{158}$ I. Maznas, ${ }^{162}$ S. M. Mazza, ${ }^{146}$ S. P. Mc Kee ${ }^{105}$ T. G. McCarthy, ${ }^{115}$ L. I. McClymont, ${ }^{94}$ W. P. McCormack, ${ }^{18}$ E. F. McDonald, ${ }^{104}$ J. A. Mcfayden, ${ }^{36}$ M. A. McKay, ${ }^{42}$ K. D. McLean, ${ }^{176}$

S. J. McMahon, ${ }^{144}$ P. C. McNamara, ${ }^{104}$ C. J. McNicol, ${ }^{178}$ R. A. McPherson, ${ }^{176, p}$ J. E. Mdhluli, ${ }^{33 c}$ Z. A. Meadows, ${ }^{102}$ S. Meehan, ${ }^{148}$ T. Megy, ${ }^{52}$ S. Mehlhase, ${ }^{114}$ A. Mehta,${ }^{90}$ T. Meideck,${ }^{58}$ B. Meirose, ${ }^{43}$ D. Melini,${ }^{174}$ B. R. Mellado Garcia,${ }^{33 c}$ J. D. Mellenthin ${ }^{53}$ M. Melo, ${ }^{28 a}$ F. Meloni, ${ }^{46}$ A. Melzer, ${ }^{24}$ S. B. Menary, ${ }^{100}$ E. D. Mendes Gouveia, ${ }^{140 a, 140 e}$ L. Meng, ${ }^{36}$ X. T. Meng, ${ }^{105}$ S. Menke, ${ }^{115}$ E. Meoni, ${ }^{41 b, 41 a}$ S. Mergelmeyer, ${ }^{19}$ S. A. M. Merkt, ${ }^{139}$ C. Merlassino, ${ }^{20}$ P. Mermod, ${ }^{54}$ L. Merola, ${ }^{69 a, 69 b}$ C. Meroni, ${ }^{68 a}$ O. Meshkov, ${ }^{13,110}$ J. K. R. Meshreki, ${ }^{151}$ A. Messina,${ }^{72 a, 72 b}$ J. Metcalfe, ${ }^{6}$ A. S. Mete, ${ }^{171}$ C. Meyer ${ }^{65}$ J. Meyer ${ }^{160}$ J-P. Meyer, ${ }^{145}$ H. Meyer Zu Theenhausen, ${ }^{61 a}$ F. Miano, ${ }^{156}$ R. P. Middleton, ${ }^{144}$ L. Mijović, ${ }^{50}$ G. Mikenberg, ${ }^{180}$ M. Mikestikova, ${ }^{141}$ M. Mikuž, ${ }^{91}$ H. Mildner, ${ }^{149}$ M. Milesi, ${ }^{104}$ A. Milic, ${ }^{167}$ D. A. Millar, ${ }^{92}$ D. W. Miller, ${ }^{37}$ 
A. Milov, ${ }^{180}$ D. A. Milstead, ${ }^{45 a, 45 b}$ R. A. Mina, ${ }^{153, e e}$ A. A. Minaenko, ${ }^{123}$ M. Miñano Moya, ${ }^{174}$ I. A. Minashvili, ${ }^{159 b}$ A. I. Mincer, ${ }^{124}$ B. Mindur, ${ }^{83 a}$ M. Mineev, ${ }^{79}$ Y. Minegishi, ${ }^{163}$ Y. Ming, ${ }^{181}$ L. M. Mir, ${ }^{14}$ A. Mirto, ${ }^{67 a, 67 b}$ K. P. Mistry, ${ }^{137}$ T. Mitani, ${ }^{179}$ J. Mitrevski, ${ }^{114}$ V. A. Mitsou, ${ }^{174}$ M. Mittal, ${ }^{60 c}$ A. Miucci, ${ }^{20}$ P. S. Miyagawa, ${ }^{149}$ A. Mizukami, ${ }^{81}$ J. U. Mjörnmark, ${ }^{96}$ T. Mkrtchyan, ${ }^{184}$ M. Mlynarikova, ${ }^{143}$ T. Moa ${ }^{45 a, 45 b}$ K. Mochizuki, ${ }^{109}$ P. Mogg,${ }^{52}$ S. Mohapatra, ${ }^{39}$ R. Moles-Valls,${ }^{24}$ M. C. Mondragon, ${ }^{106}$ K. Mönig, ${ }^{46}$ J. Monk,${ }^{40}$ E. Monnier ${ }^{101}$ A. Montalbano, ${ }^{152}$ J. Montejo Berlingen, ${ }^{36}$ M. Montella,${ }^{94}$ F. Monticelli, ${ }^{88}$ S. Monzani, ${ }^{68 a}$ N. Morange, ${ }^{132}$ D. Moreno, ${ }^{22}$ M. Moreno Llácer,${ }^{36}$ C. Moreno Martinez, ${ }^{14}$ P. Morettini, ${ }^{55 b}$ M. Morgenstern, ${ }^{120}$ S. Morgenstern, ${ }^{48}$ D. Mori, ${ }^{152}$ M. Morii ${ }^{59}$ M. Morinaga, ${ }^{179}$ V. Morisbak,${ }^{134}$ A. K. Morley ${ }^{36}$ G. Mornacchi, ${ }^{36}$ A. P. Morris, ${ }^{94}$ L. Morvaj ${ }^{155}$ P. Moschovakos, ${ }^{36}$ B. Moser, ${ }^{120}$ M. Mosidze, ${ }^{159 b}$ T. Moskalets, ${ }^{145}$ H. J. Moss, ${ }^{149}$ J. Moss, ${ }^{31, k \mathrm{k}}$ K. Motohashi, ${ }^{165}$ E. Mountricha, ${ }^{36}$ E. J. W. Moyse, ${ }^{102}$ S. Muanza, ${ }^{101}$ J. Mueller, ${ }^{139}$ R. S. P. Mueller, ${ }^{114}$ D. Muenstermann, ${ }^{89}$ G. A. Mullier, ${ }^{96}$ J. L. Munoz Martinez, ${ }^{14}$ F. J. Munoz Sanchez, ${ }^{100}$ P. Murin, ${ }^{28 b}$ W. J. Murray, ${ }^{178,144}$ A. Murrone, ${ }^{68 a, 68 b}$ M. Muškinja, ${ }^{18}$ C. Mwewa, ${ }^{33 a}$ A. G. Myagkov, ${ }^{123,11}$ J. Myers, ${ }^{131}$ M. Myska, ${ }^{142}$ B. P. Nachman, ${ }^{18}$ O. Nackenhorst, ${ }^{47}$ A. Nag Nag, ${ }^{48}$ K. Nagai,${ }^{135}$ K. Nagano, ${ }^{81}$ Y. Nagasaka, ${ }^{62}$ M. Nagel, ${ }^{52}$ E. Nagy, ${ }^{101}$ A. M. Nairz, ${ }^{36}$ Y. Nakahama,${ }^{117}$ K. Nakamura,${ }^{81}$ T. Nakamura,${ }^{163}$ I. Nakano,${ }^{127}$ H. Nanjo, ${ }^{133}$ F. Napolitano, ${ }^{61 a}$ R. F. Naranjo Garcia, ${ }^{46}$ R. Narayan, ${ }^{42}$ D. I. Narrias Villar, ${ }^{61 a}$ I. Naryshkin, ${ }^{138}$ T. Naumann, ${ }^{46}$ G. Navarro, ${ }^{22}$ H. A. Neal, ${ }^{105, a}$ P. Y. Nechaeva,${ }^{110}$ F. Nechansky, ${ }^{46}$ T. J. Neep,${ }^{21}$ A. Negri,${ }^{70 a, 70 b}$ M. Negrini, ${ }^{23 b}$ C. Nellist, ${ }^{53}$ M. E. Nelson, ${ }^{135}$ S. Nemecek, ${ }^{141}$ P. Nemethy, ${ }^{124}$ M. Nessi, ${ }^{36, m m}$ M. S. Neubauer, ${ }^{173}$ M. Neumann, ${ }^{182}$ P. R. Newman, ${ }^{21}$ T. Y. Ng ${ }^{63 c}$ Y. S. Ng, ${ }^{19}$ Y. W. Y. Ng, ${ }^{171}$ H. D. N. Nguyen, ${ }^{101}$ T. Nguyen Manh, ${ }^{109}$ E. Nibigira, ${ }^{38}$ R. B. Nickerson, ${ }^{135}$ R. Nicolaidou, ${ }^{145}$ D. S. Nielsen, ${ }^{40}$ J. Nielsen, ${ }^{146}$ N. Nikiforou, ${ }^{11}$ V. Nikolaenko, ${ }^{123,11}$ I. Nikolic-Audit, ${ }^{136}$ K. Nikolopoulos, ${ }^{21}$ P. Nilsson, ${ }^{29}$ H. R. Nindhito,${ }^{54}$ Y. Ninomiya,${ }^{81}$ A. Nisati, ${ }^{72 a}$ N. Nishu, ${ }^{60 c}$ R. Nisius, ${ }^{115}$ I. Nitsche, ${ }^{47}$ T. Nitta, ${ }^{179}$ T. Nobe ${ }^{163}$ Y. Noguchi, ${ }^{85}$ I. Nomidis, ${ }^{136}$ M. A. Nomura, ${ }^{29}$

M. Nordberg, ${ }^{36}$ N. Norjoharuddeen, ${ }^{135}$ T. Novak, ${ }^{91}$ O. Novgorodova, ${ }^{48}$ R. Novotny, ${ }^{142}$ L. Nozka, ${ }^{130}$ K. Ntekas, ${ }^{171}$ E. Nurse, ${ }^{94}$ F. G. Oakham, ${ }^{34, e}$ H. Oberlack, ${ }^{115}$ J. Ocariz, ${ }^{136}$ A. Ochi, ${ }^{82}$ I. Ochoa, ${ }^{39}$ J. P. Ochoa-Ricoux, ${ }^{147 a}$ K. O'Connor, ${ }^{26}$ S. Oda ${ }^{87}$ S. Odaka, ${ }^{81}$ S. Oerdek, ${ }^{53}$ A. Ogrodnik ${ }^{83 a}$ A. Oh ${ }^{100}$ S. H. Oh ${ }^{49}$ C. C. Ohm, ${ }^{154}$ H. Oide, ${ }^{55 b 55 a}$ M. L. Ojeda, ${ }^{167}$ H. Okawa, ${ }^{169}$ Y. Okazaki, ${ }^{85}$ Y. Okumura, ${ }^{163}$ T. Okuyama, ${ }^{81}$ A. Olariu, ${ }^{27 b}$ L. F. Oleiro Seabra, ${ }^{140 a}$ S. A. Olivares Pino, ${ }^{147 a}$ D. Oliveira Damazio, ${ }^{29}$ J. L. Oliver, ${ }^{1}$ M. J. R. Olsson, ${ }^{171}$ A. Olszewski, ${ }^{84}$ J. Olszowska, ${ }^{84}$ D. C. O'Neil, ${ }^{152}$ A. Onofre, ${ }^{140 a, 140 e}$ K. Onogi, ${ }^{117}$ P. U. E. Onyisi, ${ }^{11}$ H. Oppen, ${ }^{134}$ M. J. Oreglia, ${ }^{37}$ G. E. Orellana, ${ }^{88}$ Y. Oren, ${ }^{161}$ D. Orestano, ${ }^{74 a, 74 b}$ N. Orlando, ${ }^{14}$ R. S. Orr, ${ }^{167}$ V. O’Shea, ${ }^{57}$ R. Ospanov, ${ }^{60 a}$ G. Otero y Garzon, ${ }^{30}$ H. Otono, ${ }^{87}$ M. Ouchrif, ${ }^{35 d}$ J. Ouellette, ${ }^{29}$ F. Ould-Saada, ${ }^{134}$ A. Ouraou, ${ }^{145}$ Q. Ouyang, ${ }^{15 a}$ M. Owen,${ }^{57}$ R. E. Owen, ${ }^{21}$ V. E. Ozcan, ${ }^{12 c}$ N. Ozturk, ${ }^{8}$ J. Pacalt, ${ }^{130}$ H. A. Pacey, ${ }^{32}$ K. Pachal,${ }^{49}$ A. Pacheco Pages, ${ }^{14}$ C. Padilla Aranda,${ }^{14}$ S. Pagan Griso,${ }^{18}$ M. Paganini, ${ }^{183}$ G. Palacino, ${ }^{65}$ S. Palazzo,${ }^{50}$ S. Palestini, ${ }^{36}$ M. Palka, ${ }^{83 b}$ D. Pallin, ${ }^{38}$ I. Panagoulias, ${ }^{10}$ C. E. Pandini, ${ }^{36}$ J. G. Panduro Vazquez, ${ }^{93}$ P. Pani, ${ }^{46}$ G. Panizzo, ${ }^{66 a, 66 c}$

L. Paolozzi, ${ }^{54}$ C. Papadatos, ${ }^{109}$ K. Papageorgiou, ${ }^{9, s}$ A. Paramonov, ${ }^{6}$ D. Paredes Hernandez, ${ }^{63 b}$ S. R. Paredes Saenz, ${ }^{135}$ B. Parida, ${ }^{166}$ T. H. Park, ${ }^{167}$ A. J. Parker ${ }^{89}$ M. A. Parker, ${ }^{32}$ F. Parodi, ${ }^{55 b, 55 a}$ E. W. P. Parrish, ${ }^{121}$ J. A. Parsons, ${ }^{39}$ U. Parzefall, ${ }^{52}$ L. Pascual Dominguez, ${ }^{136}$ V. R. Pascuzzi, ${ }^{167}$ J. M. P. Pasner, ${ }^{146}$ E. Pasqualucci ${ }^{72 a}$ S. Passaggio, ${ }^{55 b}$ F. Pastore, ${ }^{93}$ P. Pasuwan, ${ }^{45 a, 45 b}$ S. Pataraia, ${ }^{99}$ J. R. Pater ${ }^{100}$ A. Pathak, ${ }^{181}$ T. Pauly,${ }^{36}$ B. Pearson, ${ }^{115}$ M. Pedersen, ${ }^{134}$ L. Pedraza Diaz, ${ }^{119}$ R. Pedro, ${ }^{140 a}$ T. Peiffer, ${ }^{53}$ S. V. Peleganchuk, ${ }^{122 b, 122 a}$ O. Penc, ${ }^{141}$ H. Peng, ${ }^{60 a}$ B. S. Peralva, ${ }^{80 a}$ M. M. Perego, ${ }^{132}$ A. P. Pereira Peixoto, ${ }^{140 \mathrm{a}}$ D. V. Perepelitsa, ${ }^{29}$ F. Peri, ${ }^{19}$ L. Perini, ${ }^{68 \mathrm{a}, 68 \mathrm{~b}}$ H. Pernegger, ${ }^{36}$ S. Perrella, ${ }^{69 a, 69 \mathrm{~b}}$ K. Peters,${ }^{46}$ R. F. Y. Peters, ${ }^{100}$ B. A. Petersen, ${ }^{36}$ T. C. Petersen,${ }^{40}$ E. Petit, ${ }^{101}$ A. Petridis, ${ }^{1}$ C. Petridou, ${ }^{162}$ P. Petroff, ${ }^{132}$ M. Petrov, ${ }^{135}$ F. Petrucci, ${ }^{74 a, 74 b}$ M. Pettee, ${ }^{183}$ N. E. Pettersson, ${ }^{102}$ K. Petukhova, ${ }^{143}$ A. Peyaud ${ }^{145}$ R. Pezoa,${ }^{147 b}$ L. Pezzotti, ${ }^{70 a, 70 b}$

T. Pham, ${ }^{104}$ F. H. Phillips,${ }^{106}$ P. W. Phillips, ${ }^{144}$ M. W. Phipps,${ }^{173}$ G. Piacquadio, ${ }^{155}$ E. Pianori, ${ }^{18}$ A. Picazio, ${ }^{102}$ R. H. Pickles, ${ }^{100}$ R. Piegaia, ${ }^{30}$ D. Pietreanu, ${ }^{27 b}$ J. E. Pilcher, ${ }^{37}$ A. D. Pilkington, ${ }^{100}$ M. Pinamonti, ${ }^{73 a, 73 b}$ J. L. Pinfold, ${ }^{3}$ M. Pitt, ${ }^{180}$ L. Pizzimento, ${ }^{73 a, 73 b}$ M.-A. Pleier, ${ }^{29}$ V. Pleskot, ${ }^{143}$ E. Plotnikova, ${ }^{79}$ D. Pluth ${ }^{78}$ P. Podberezko, ${ }^{122 b, 122 a}$ R. Poettgen, ${ }^{96}$ R. Poggi, ${ }^{54}$ L. Poggioli, ${ }^{132}$ I. Pogrebnyak, ${ }^{106}$ D. Pohl,${ }^{24}$ I. Pokharel,${ }^{53}$ G. Polesello, ${ }^{70 a}$ A. Poley, ${ }^{18}$ A. Policicchio, ${ }^{72 a, 72 b}$ R. Polifka, ${ }^{143}$ A. Polini, ${ }^{23 b}$ C. S. Pollard, ${ }^{46}$ V. Polychronakos ${ }^{29}$ D. Ponomarenko, ${ }^{112}$ L. Pontecorvo, ${ }^{36}$ S. Popa ${ }^{27 a}$ G. A. Popeneciu, ${ }^{27 d}$ D. M. Portillo Quintero, ${ }^{58}$ S. Pospisil, ${ }^{142}$ K. Potamianos, ${ }^{46}$ I. N. Potrap, ${ }^{79}$ C. J. Potter, ${ }^{32}$ H. Potti, ${ }^{11}$ T. Poulsen, ${ }^{96}$ J. Poveda,${ }^{36}$ T. D. Powell, ${ }^{149}$ G. Pownall, ${ }^{46}$ M. E. Pozo Astigarraga,${ }^{36}$ P. Pralavorio, ${ }^{101}$ S. Prell,${ }^{78}$ D. Price, ${ }^{100}$ M. Primavera, ${ }^{67 a}$ S. Prince ${ }^{103}$ M. L. Proffitt, ${ }^{148}$ N. Proklova, ${ }^{112}$ K. Prokofiev,${ }^{63 c}$ F. Prokoshin, ${ }^{79}$ S. Protopopescu, ${ }^{29}$ J. Proudfoot, ${ }^{6}$ M. Przybycien, ${ }^{83 a}$ D. Pudzha, ${ }^{138}$ A. Puri, ${ }^{173}$ P. Puzo, ${ }^{132}$ J. Qian, ${ }^{105}$ Y. Qin, ${ }^{100}$ A. Quadt,${ }^{53}$ M. Queitsch-Maitland, ${ }^{46}$ A. Qureshi, ${ }^{1}$ P. Rados,${ }^{104}$ F. Ragusa, ${ }^{68 a}, 68 b$ G. Rahal,${ }^{97}$ J. A. Raine, ${ }^{54}$ S. Rajagopalan, ${ }^{29}$ A. Ramirez Morales, ${ }^{92}$ K. Ran, ${ }^{15 a, 15 \mathrm{~d}}$ T. Rashid, ${ }^{132}$ S. Raspopov, ${ }^{5}$ M. G. Ratti ${ }^{68 \mathrm{a}, 68 \mathrm{~b}}$ D. M. Rauch, ${ }^{46}$ F. Rauscher, ${ }^{114}$ S. Rave, ${ }^{99}$ B. Ravina,${ }^{149}$ I. Ravinovich, ${ }^{180}$ J. H. Rawling, ${ }^{100}$ M. Raymond, ${ }^{36}$ A. L. Read, ${ }^{134}$ N. P. Readioff, ${ }^{58}$ M. Reale, ${ }^{67 a, 67 b}$ 
D. M. Rebuzzi, ${ }^{70 a, 70 b}$ A. Redelbach, ${ }^{177}$ G. Redlinger, ${ }^{29}$ K. Reeves,${ }^{43}$ L. Rehnisch, ${ }^{19}$ J. Reichert, ${ }^{137}$ D. Reikher, ${ }^{161}$ A. Reiss, ${ }^{99}$ A. Rej, ${ }^{151}$ C. Rembser, ${ }^{36}$ M. Renda, ${ }^{27 b}$ M. Rescigno, ${ }^{72 a}$ S. Resconi, ${ }^{68 a}$ E. D. Resseguie, ${ }^{137}$ S. Rettie ${ }^{175}$ E. Reynolds,${ }^{21}$ O. L. Rezanova, ${ }^{122 b, 122 a}$ P. Reznicek, ${ }^{143}$ E. Ricci ${ }^{75 a, 75 b}$ R. Richter, ${ }^{115}$ S. Richter, ${ }^{46}$ E. Richter-Was, ${ }^{83 b}$ O. Ricken, ${ }^{24}$

M. Ridel, ${ }^{136}$ P. Rieck, ${ }^{115}$ C. J. Riegel, ${ }^{182}$ O. Rifki, ${ }^{46}$ M. Rijssenbeek, ${ }^{155}$ A. Rimoldi, ${ }^{70 a, 70 b}$ M. Rimoldi, ${ }^{46}$ L. Rinaldi, ${ }^{23 b}$ G. Ripellino, ${ }^{154}$ B. Ristić, ${ }^{89}$ E. Ritsch,${ }^{36}$ I. Riu, ${ }^{14}$ J. C. Rivera Vergara, ${ }^{176}$ F. Rizatdinova, ${ }^{129}$ E. Rizvi, ${ }^{92}$ C. Rizzi ${ }^{36}$ R. T. Roberts, ${ }^{100}$ S. H. Robertson, ${ }^{103, p}$ M. Robin, ${ }^{46}$ D. Robinson, ${ }^{32}$ J. E. M. Robinson, ${ }^{46}$ C. M. Robles Gajardo, ${ }^{147 b}$ A. Robson,${ }^{57}$ E. Rocco, ${ }^{99}$ C. Roda,${ }^{71 a, 71 b}$ S. Rodriguez Bosca,${ }^{174}$ A. Rodriguez Perez, ${ }^{14}$ D. Rodriguez Rodriguez,${ }^{174}$ A. M. Rodríguez Vera, ${ }^{168 b}$ S. Roe, ${ }^{36}$ O. Røhne, ${ }^{134}$ R. Röhrig, ${ }^{115}$ C. P. A. Roland, ${ }^{65}$ J. Roloff, ${ }^{59}$ A. Romaniouk, ${ }^{112}$ M. Romano, ${ }^{23 b, 23 a}$ N. Rompotis, ${ }^{90}$ M. Ronzani, ${ }^{124}$ L. Roos, ${ }^{136}$ S. Rosati, ${ }^{72 a}$ K. Rosbach,${ }^{52}$ G. Rosin, ${ }^{102}$ B. J. Rosser, ${ }^{137}$ E. Rossi ${ }^{46}$ E. Rossi, ${ }^{74 a, 74 b}$ E. Rossi, ${ }^{69 a, 69 b}$ L. P. Rossi,${ }^{55 b}$ L. Rossini, ${ }^{68,68 b}$ R. Rosten, ${ }^{14}$ M. Rotaru, ${ }^{27 b}$ J. Rothberg, ${ }^{148}$ D. Rousseau, ${ }^{132}$ G. Rovelli, ${ }^{70 a, 70 b}$ D. Roy, ${ }^{33 c}$ A. Rozanov, ${ }^{101}$ Y. Rozen, ${ }^{160}$ X. Ruan, ${ }^{33 c}$ F. Rubbo, ${ }^{153}$ F. Rühr, ${ }^{52}$ A. Ruiz-Martinez, ${ }^{174}$ A. Rummler, ${ }^{36}$ Z. Rurikova ${ }^{52}$ N. A. Rusakovich, ${ }^{79}$ H. L. Russell, ${ }^{103}$ L. Rustige, ${ }^{38,47}$ J. P. Rutherfoord, ${ }^{7}$ E. M. Rüttinger, ${ }^{46, n n}$ Y. F. Ryabov, ${ }^{138}$ M. Rybar, ${ }^{39}$ G. Rybkin, ${ }^{132}$ A. Ryzhov, ${ }^{123}$ G. F. Rzehorz, ${ }^{53}$ P. Sabatini, ${ }^{53}$ G. Sabato, ${ }^{120}$ S. Sacerdoti, ${ }^{132}$ H. F-W. Sadrozinski, ${ }^{146}$ R. Sadykov, ${ }^{79}$ F. Safai Tehrani, ${ }^{72 a}$ B. Safarzadeh Samani, ${ }^{156}$ P. Saha, ${ }^{121}$ S. Saha, ${ }^{103}$ M. Sahinsoy, ${ }^{61 \mathrm{a}}$ A. Sahu, ${ }^{182}$ M. Saimpert, ${ }^{46}$ M. Saito, ${ }^{163}$ T. Saito, ${ }^{163}$ H. Sakamoto, ${ }^{163}$ A. Sakharov, ${ }^{124, f f}$ D. Salamani, ${ }^{54}$ G. Salamanna, ${ }^{74 a, 74 b}$ J. E. Salazar Loyola, ${ }^{147 b}$ P. H. Sales De Bruin, ${ }^{172}$ A. Salnikov, ${ }^{153}$ J. Salt, ${ }^{174}$ D. Salvatore, ${ }^{41 b, 41 a}$ F. Salvatore, ${ }^{156}$ A. Salvucci, ${ }^{63 a, 63 b, 63 c}$ A. Salzburger, ${ }^{36}$ J. Samarati, ${ }^{36}$ D. Sammel,${ }^{52}$ D. Sampsonidis, ${ }^{162}$ D. Sampsonidou, ${ }^{162}$ J. Sánchez, ${ }^{174}$ A. Sanchez Pineda,${ }^{66 a, 66 c}$ H. Sandaker, ${ }^{134}$ C. O. Sander, ${ }^{46}$ I. G. Sanderswood, ${ }^{89}$ M. Sandhoff, ${ }^{182}$

C. Sandoval, ${ }^{22}$ D. P. C. Sankey, ${ }^{144}$ M. Sannino,${ }^{55 b, 55 a}$ Y. Sano, ${ }^{117}$ A. Sansoni, ${ }^{51}$ C. Santoni,${ }^{38}$ H. Santos, ${ }^{140 a, 140 b}$ S. N. Santpur, ${ }^{18}$ A. Santra, ${ }^{174}$ A. Sapronov, ${ }^{79}$ J. G. Saraiva, ${ }^{140 a, 140 d}$ O. Sasaki, ${ }^{81}$ K. Sato, ${ }^{169}$ E. Sauvan, ${ }^{5}$ P. Savard, ${ }^{167, e}$ N. Savic, ${ }^{115}$ R. Sawada, ${ }^{163}$ C. Sawyer, ${ }^{144}$ L. Sawyer, ${ }^{95,00}$ C. Sbarra, ${ }^{23 b}$ A. Sbrizzi, ${ }^{23 a}$ T. Scanlon, ${ }^{94}$ J. Schaarschmidt, ${ }^{148}$ P. Schacht, ${ }^{115}$ B. M. Schachtner, ${ }^{114}$ D. Schaefer, ${ }^{37}$ L. Schaefer, ${ }^{137}$ J. Schaeffer,${ }^{99}$ S. Schaepe,${ }^{36}$ U. Schäfer, ${ }^{99}$ A. C. Schaffer, ${ }^{132}$ D. Schaile, ${ }^{114}$ R. D. Schamberger, ${ }^{155}$ N. Scharmberg, ${ }^{100}$ V. A. Schegelsky, ${ }^{138}$ D. Scheirich, ${ }^{143}$ F. Schenck, ${ }^{19}$ M. Schernau, ${ }^{171}$ C. Schiavi, ${ }^{55 b, 55 a}$ S. Schier ${ }^{146}$ L. K. Schildgen, ${ }^{24}$ Z. M. Schillaci, ${ }^{26}$ E. J. Schioppa ${ }^{36}$ M. Schioppa ${ }^{41 b, 41 a}$ K. E. Schleicher, ${ }^{52}$ S. Schlenker, ${ }^{36}$ K. R. Schmidt-Sommerfeld, ${ }^{115}$ K. Schmieden, ${ }^{36}$ C. Schmitt, ${ }^{99}$ S. Schmitt, ${ }^{46}$ S. Schmitz, ${ }^{99}$ J. C. Schmoeckel, ${ }^{46}$ U. Schnoor, ${ }^{52}$ L. Schoeffel, ${ }^{145}$ A. Schoening, ${ }^{61 b}$ P. G. Scholer, ${ }^{52}$ E. Schopf ${ }^{135}$ M. Schott, ${ }^{99}$ J. F. P. Schouwenberg, ${ }^{119}$ J. Schovancova, ${ }^{36}$ S. Schramm, ${ }^{54}$ F. Schroeder, ${ }^{182}$ A. Schulte, ${ }^{99}$ H-C. Schultz-Coulon, ${ }^{61 a}$ M. Schumacher, ${ }^{52}$ B. A. Schumm, ${ }^{146}$ Ph. Schune, ${ }^{145}$ A. Schwartzman, ${ }^{153}$ T. A. Schwarz, ${ }^{105}$ Ph. Schwemling, ${ }^{145}$ R. Schwienhorst, ${ }^{106}$ A. Sciandra, ${ }^{146}$ G. Sciolla, ${ }^{26}$ M. Scodeggio, ${ }^{46}$ M. Scornajenghi,${ }^{41 b, 41 a}$ F. Scuri, ${ }^{71 a}$ F. Scutti, ${ }^{104}$ L. M. Scyboz, ${ }^{115}$ C. D. Sebastiani, ${ }^{72 a, 72 b}$ P. Seema, ${ }^{19}$ S. C. Seidel, ${ }^{118}$ A. Seiden, ${ }^{146}$ T. Seiss, ${ }^{37}$ J. M. Seixas, ${ }^{80 b}$ G. Sekhniaidze, ${ }^{69 a}$ K. Sekhon, ${ }^{105}$ S. J. Sekula, ${ }^{42}$ N. Semprini-Cesari, ${ }^{23 b, 23 a}$ S. Sen, ${ }^{49}$ S. Senkin, ${ }^{38}$ C. Serfon, ${ }^{76}$ L. Serin, ${ }^{132}$ L. Serkin, ${ }^{66 a, 66 b}$ M. Sessa, ${ }^{60 a}$ H. Severini, ${ }^{128}$ F. Sforza, ${ }^{170}$ A. Sfyrla, ${ }^{54}$ E. Shabalina, ${ }^{53}$ J. D. Shahinian, ${ }^{146}$ N. W. Shaikh, ${ }^{45 a, 45 b}$ D. Shaked Renous, ${ }^{180}$ L. Y. Shan, ${ }^{15 a}$ R. Shang, ${ }^{173}$ J. T. Shank, ${ }^{25}$ M. Shapiro, ${ }^{18}$ A. Sharma, ${ }^{135}$ A. S. Sharma, ${ }^{1}$ P. B. Shatalov, ${ }^{111}$ K. Shaw, ${ }^{156}$ S. M. Shaw,${ }^{100}$ A. Shcherbakova, ${ }^{138}$ Y. Shen,${ }^{128}$ N. Sherafati, ${ }^{34}$ A. D. Sherman, ${ }^{25}$ P. Sherwood, ${ }^{94}$ L. Shi, ${ }^{158, p p}$ S. Shimizu, ${ }^{81}$ C. O. Shimmin, ${ }^{183}$ Y. Shimogama, ${ }^{179}$ M. Shimojima, ${ }^{116}$ I. P. J. Shipsey, ${ }^{135}$ S. Shirabe, ${ }^{87}$ M. Shiyakova, ${ }^{79, q q}$ J. Shlomi, ${ }^{180}$ A. Shmeleva, ${ }^{110}$ M. J. Shochet,${ }^{37}$ S. Shojaii, ${ }^{104}$ D. R. Shope,${ }^{128}$ S. Shrestha, ${ }^{126}$ E. Shulga, ${ }^{180}$ P. Sicho, ${ }^{141}$ A. M. Sickles, ${ }^{173}$ P. E. Sidebo, ${ }^{154}$ E. Sideras Haddad, ${ }^{33 c}$ O. Sidiropoulou, ${ }^{36}$ A. Sidoti, ${ }^{23 b, 23 a}$

F. Siegert, ${ }^{48}$ Dj. Sijacki, ${ }^{16}$ M. Silva Jr., ${ }^{181}$ M. V. Silva Oliveira, ${ }^{80 a}$ S. B. Silverstein, ${ }^{45 a}$ S. Simion, ${ }^{132}$ E. Simioni, ${ }^{99}$ R. Simoniello, ${ }^{99}$ S. Simsek, ${ }^{12 b}$ P. Sinervo, ${ }^{167}$ V. Sinetckii, ${ }^{113,110}$ N. B. Sinev, ${ }^{131}$ M. Sioli,${ }^{23 b, 23 a}$ I. Siral, ${ }^{105}$

S. Yu. Sivoklokov, ${ }^{113}$ J. Sjölin, ${ }^{45 a, 45 b}$ E. Skorda,${ }^{96}$ P. Skubic, ${ }^{128}$ M. Slawinska,${ }^{84}$ K. Sliwa ${ }^{170}$ R. Slovak, ${ }^{143}$ V. Smakhtin, ${ }^{180}$ B. H. Smart, ${ }^{144}$ J. Smiesko, ${ }^{28 a}$ N. Smirnov, ${ }^{112}$ S. Yu. Smirnov, ${ }^{112}$ Y. Smirnov, ${ }^{112}$ L. N. Smirnova, ${ }^{11, \text { rr }}$ O. Smirnova, ${ }^{96}$ J. W. Smith, ${ }^{53}$ M. Smizanska, ${ }^{89}$ K. Smolek, ${ }^{142}$ A. Smykiewicz, ${ }^{84}$ A. A. Snesarev, ${ }^{110}$ H. L. Snoek, ${ }^{120}$ I. M. Snyder, ${ }^{131}$ S. Snyder, ${ }^{29}$ R. Sobie, ${ }^{176, p}$ A. M. Soffa, ${ }^{171}$ A. Soffer,${ }^{161}$ A. Søgaard,${ }^{50}$ F. Sohns,${ }^{53}$ C. A. Solans Sanchez, ${ }^{36}$ E. Yu. Soldatov, ${ }^{112}$ U. Soldevila, ${ }^{174}$ A. A. Solodkov, ${ }^{123}$ A. Soloshenko, ${ }^{79}$ O. V. Solovyanov, ${ }^{123}$ V. Solovyev, ${ }^{138}$ P. Sommer, ${ }^{149}$ H. Son, ${ }^{170}$ W. Song, ${ }^{144}$ W. Y. Song, ${ }^{168 b}$ A. Sopczak, ${ }^{142}$ F. Sopkova, ${ }^{28 b}$ C. L. Sotiropoulou, ${ }^{71 a, 71 b}$ S. Sottocornola ${ }^{70 a, 70 b}$ R. Soualah, ${ }^{66 a, 66 c, s s}$ A. M. Soukharev, ${ }^{122 b, 122 a}$ D. South, ${ }^{46}$ S. Spagnolo,${ }^{67 a, 67 b}$ M. Spalla, ${ }^{115}$ M. Spangenberg, ${ }^{178}$ F. Spanò,${ }^{93}$ D. Sperlich, ${ }^{52}$ T. M. Spieker, ${ }^{61 \mathrm{a}}$ R. Spighi, ${ }^{23 \mathrm{~b}}$ G. Spigo, ${ }^{36}$ M. Spina,${ }^{156}$ D. P. Spiteri, ${ }^{57}$ M. Spousta, ${ }^{143}$ A. Stabile, ${ }^{68 a, 68 \mathrm{~b}}$ B. L. Stamas, ${ }^{121}$ R. Stamen, ${ }^{61 a}$ M. Stamenkovic, ${ }^{120}$ E. Stanecka, ${ }^{84}$ R. W. Stanek, ${ }^{6}$ B. Stanislaus, ${ }^{135}$ M. M. Stanitzki, ${ }^{46}$ M. Stankaityte, ${ }^{135}$ B. Stapf, ${ }^{120}$ E. A. Starchenko, ${ }^{123}$ G. H. Stark, ${ }^{146}$ J. Stark,${ }^{58}$ S. H Stark, ${ }^{40}$ P. Staroba, ${ }^{141}$ P. Starovoitov, ${ }^{61 a}$ 
S. Stärz, ${ }^{103}$ R. Staszewski, ${ }^{84}$ G. Stavropoulos, ${ }^{44}$ M. Stegler, ${ }^{46}$ P. Steinberg, ${ }^{29}$ A. L. Steinhebel, ${ }^{131}$ B. Stelzer, ${ }^{152}$ H. J. Stelzer, ${ }^{139}$ O. Stelzer-Chilton, ${ }^{168 a}$ H. Stenzel,${ }^{56}$ T. J. Stevenson, ${ }^{156}$ G. A. Stewart, ${ }^{36}$ M. C. Stockton,${ }^{36}$ G. Stoicea, ${ }^{27 b}$ M. Stolarski, ${ }^{140 a}$ P. Stolte, ${ }^{53}$ S. Stonjek, ${ }^{115}$ A. Straessner, ${ }^{48}$ J. Strandberg, ${ }^{154}$ S. Strandberg, $,{ }^{45 a}, 45 \mathrm{~b}$ M. Strauss, ${ }^{128}$ P. Strizenec, ${ }^{28 b}$ R. Ströhmer, ${ }^{177}$ D. M. Strom, ${ }^{131}$ R. Stroynowski, ${ }^{42}$ A. Strubig, ${ }^{50}$ S. A. Stucci, ${ }^{29}$ B. Stugu, ${ }^{17}$ J. Stupak, ${ }^{128}$ N. A. Styles, ${ }^{46}$ D. Su, ${ }^{153}$ S. Suchek, ${ }^{61 a}$ V. V. Sulin, ${ }^{110}$ M. J. Sullivan, ${ }^{90}$ D. M. S. Sultan, ${ }^{54}$ S. Sultansoy, ${ }^{4 c}$ T. Sumida ${ }^{85}$ S. Sun, ${ }^{105}$ X. Sun, ${ }^{3}$ K. Suruliz, ${ }^{156}$ C. J. E. Suster, ${ }^{157}$ M. R. Sutton, ${ }^{156}$ S. Suzuki, ${ }^{81}$ M. Svatos, ${ }^{141}$ M. Swiatlowski ${ }^{37}$ S. P. Swift, ${ }^{2}$ T. Swirski, ${ }^{177}$ A. Sydorenko, ${ }^{99}$ I. Sykora ${ }^{28 a}$ M. Sykora, ${ }^{143}$ T. Sykora,${ }^{143}$ D. Ta, ${ }^{99}$ K. Tackmann, ${ }^{46, t t}$ J. Taenzer, ${ }^{161}$ A. Taffard, ${ }^{171}$ R. Tafirout, ${ }^{168}$ H. Takai, ${ }^{29}$ R. Takashima, ${ }^{86}$ K. Takeda,${ }^{82}$ T. Takeshita, ${ }^{150}$ E. P. Takeva ${ }^{50}$ Y. Takubo, ${ }^{81}$ M. Talby, ${ }^{101}$ A. A. Talyshev, ${ }^{122 b, 122 a}$ N. M. Tamir, ${ }^{161}$ J. Tanaka, ${ }^{163}$ M. Tanaka, ${ }^{165}$ R. Tanaka, ${ }^{132}$ S. Tapia Araya, ${ }^{173}$ S. Tapprogge, ${ }^{99}$ A. Tarek Abouelfadl Mohamed, ${ }^{136}$ S. Tarem, ${ }^{160}$ G. Tarna, ${ }^{27 b, u u}$ G. F. Tartarelli, ${ }^{68 a}$ P. Tas, ${ }^{143}$ M. Tasevsky, ${ }^{141}$ T. Tashiro, ${ }^{85}$ E. Tassi ${ }^{41 \mathrm{~b}, 41 \mathrm{a}}$ A. Tavares Delgado, ${ }^{140 \mathrm{a}, 140 \mathrm{~b}}$ Y. Tayalati, ${ }^{35 \mathrm{e}}$ A. J. Taylor, ${ }^{50}$ G. N. Taylor, ${ }^{104}$ W. Taylor, ${ }^{168 \mathrm{~b}}$ A. S. Tee ${ }^{89}$ R. Teixeira De Lima, ${ }^{153}$ P. Teixeira-Dias, ${ }^{93}$ H. Ten Kate, ${ }^{36}$ J. J. Teoh, ${ }^{120}$ S. Terada, ${ }^{81} \mathrm{~K}$. Terashi, ${ }^{163}$ J. Terron, ${ }^{98}$

S. Terzo, ${ }^{14}$ M. Testa, ${ }^{51}$ R. J. Teuscher, ${ }^{167, p}$ S. J. Thais ${ }^{183}$ T. Theveneaux-Pelzer, ${ }^{46}$ F. Thiele, ${ }^{40}$ D. W. Thomas, ${ }^{93}$ J. O. Thomas, ${ }^{42}$ J. P. Thomas, ${ }^{21}$ A. S. Thompson,${ }^{57}$ P. D. Thompson, ${ }^{21}$ L. A. Thomsen, ${ }^{183}$ E. Thomson, ${ }^{137}$ Y. Tian,${ }^{39}$ R. E. Ticse Torres ${ }^{53}$ V. O. Tikhomirov, ${ }^{10, v v}$ Yu. A. Tikhonov, ${ }^{122 b, 122 a}$ S. Timoshenko, ${ }^{112}$ P. Tipton, ${ }^{183}$ S. Tisserant,${ }^{101}$ K. Todome, ${ }^{23 b, 23 a}$ S. Todorova-Nova,${ }^{5}$ S. Todt, ${ }^{48}$ J. Tojo, ${ }^{87}$ S. Tokár, ${ }^{28 a}$ K. Tokushuku, ${ }^{81}$ E. Tolley, ${ }^{126}$ K. G. Tomiwa, ${ }^{33 c}$ M. Tomoto, ${ }^{117}$ L. Tompkins, ${ }^{153, e e}$ K. Toms,${ }^{118}$ B. Tong,${ }^{59}$ P. Tornambe, ${ }^{102}$ E. Torrence,${ }^{131}$ H. Torres, ${ }^{48}$ E. Torró Pastor, ${ }^{148}$ C. Tosciri, ${ }^{135}$ J. Toth, ${ }^{101, w w}$ D. R. Tovey, ${ }^{149}$ A. Traeet, ${ }^{17}$ C. J. Treado, ${ }^{124}$ T. Trefzger, ${ }^{177}$ F. Tresoldi, ${ }^{156}$ A. Tricoli, ${ }^{29}$ I. M. Trigger, ${ }^{168 \mathrm{a}}$ S. Trincaz-Duvoid, ${ }^{136} \mathrm{~W}$. Trischuk, ${ }^{167}$ B. Trocmé, ${ }^{58}$ A. Trofymov, ${ }^{132} \mathrm{C}$. Troncon, ${ }^{68 \mathrm{a}} \mathrm{M}$. Trovatelli, ${ }^{176}$ F. Trovato, ${ }^{156}$ L. Truong, ${ }^{33 b}$ M. Trzebinski,${ }^{84}$ A. Trzupek ${ }^{84}$ F. Tsai, ${ }^{46}$ J. C-L. Tseng, ${ }^{135}$ P. V. Tsiareshka, ${ }^{107, g g}$ A. Tsirigotis, ${ }^{162}$ N. Tsirintanis, ${ }^{9}$ V. Tsiskaridze, ${ }^{155}$ E. G. Tskhadadze, ${ }^{159 a}$ M. Tsopoulou, ${ }^{162}$ I. I. Tsukerman, ${ }^{111}$ V. Tsulaia, ${ }^{18}$ S. Tsuno, ${ }^{81}$

D. Tsybychev, ${ }^{155}$ Y. Tu, ${ }^{63 b}$ A. Tudorache, ${ }^{27 b}$ V. Tudorache, ${ }^{27 b}$ T. T. Tulbure, ${ }^{27 a}$ A. N. Tuna, ${ }^{59}$ S. Turchikhin, ${ }^{79}$

D. Turgeman, ${ }^{180}$ I. Turk Cakir, ${ }^{4 b, x x}$ R. J. Turner ${ }^{21}$ R. T. Turra, ${ }^{68 a}$ P. M. Tuts, ${ }^{39}$ S Tzamarias, ${ }^{162}$ E. Tzovara, ${ }^{99}$ G. Ucchielli, ${ }^{47}$ K. Uchida, ${ }^{163}$ I. Ueda, ${ }^{81}$ M. Ughetto, ${ }^{45 a, 45 b}$ F. Ukegawa, ${ }^{169}$ G. Unal, ${ }^{36}$ A. Undrus, ${ }^{29}$ G. Unel, ${ }^{171}$ F. C. Ungaro, ${ }^{104}$ Y. Unno, ${ }^{81}$ K. Uno, ${ }^{163}$ J. Urban, ${ }^{28 b}$ P. Urquijo, ${ }^{104}$ G. Usai, ${ }^{8}$ J. Usui, ${ }^{81}$ Z. Uysal, ${ }^{12 \mathrm{~d}}$ L. Vacavant, ${ }^{101}$ V. Vacek, ${ }^{142}$ B. Vachon, ${ }^{103}$ K. O. H. Vadla, ${ }^{134}$ A. Vaidya, ${ }^{94}$ C. Valderanis, ${ }^{114}$ E. Valdes Santurio, ${ }^{45 a, 45 b}$ M. Valente, ${ }^{54}$ S. Valentinetti, ${ }^{23 b, 23 a}$ A. Valero, ${ }^{174}$ L. Valéry, ${ }^{46}$ R. A. Vallance, ${ }^{21}$ A. Vallier,${ }^{36}$ J. A. Valls Ferrer, ${ }^{174}$ T. R. Van Daalen, ${ }^{14}$ P. Van Gemmeren, ${ }^{6}$ I. Van Vulpen, ${ }^{120}$ M. Vanadia, ${ }^{73 a, 73 b}$ W. Vandelli, ${ }^{36}$ A. Vaniachine, ${ }^{166}$ D. Vannicola, ${ }^{72 a, 72 b}$ R. Vari, ${ }^{72 a}$ E. W. Varnes, ${ }^{7}$ C. Varni, ${ }^{55 b, 55 a}$ T. Varol, ${ }^{42}$ D. Varouchas, ${ }^{132}$ K. E. Varvell, ${ }^{157}$ M. E. Vasile, ${ }^{27 b}$ G. A. Vasquez, ${ }^{176}$ J. G. Vasquez, ${ }^{183}$ F. Vazeille, ${ }^{38}$ D. Vazquez Furelos, ${ }^{14}$ T. Vazquez Schroeder, ${ }^{36}$ J. Veatch, ${ }^{53}$ V. Vecchio, ${ }^{74 a, 74 b}$ M. J. Veen, ${ }^{120}$ L. M. Veloce, ${ }^{167}$ F. Veloso, ${ }^{140 a, 140 \mathrm{c}}$ S. Veneziano, ${ }^{72 a}$ A. Ventura, ${ }^{67,67 b}$ N. Venturi, ${ }^{36}$ A. Verbytskyi, ${ }^{115}$ V. Vercesi, ${ }^{70 a}$ M. Verducci, ${ }^{74 a, 74 b}$ C. M. Vergel Infante, ${ }^{78}$ C. Vergis, ${ }^{24}$ W. Verkerke, ${ }^{120}$ A. T. Vermeulen, ${ }^{120}$ J. C. Vermeulen, ${ }^{120}$ M. C. Vetterli, ${ }^{152, \mathrm{e}}$ N. Viaux Maira ${ }^{147 \mathrm{~b}}$ M. Vicente Barreto Pinto,${ }^{54}$ T. Vickey, ${ }^{149}$ O. E. Vickey Boeriu, ${ }^{149}$ G. H. A. Viehhauser, ${ }^{135}$ L. Vigani ${ }^{135}$ M. Villa, ${ }^{23 b, 23 a}$ M. Villaplana Perez, ${ }^{68 a, 68 b}$ E. Vilucchi, ${ }^{51}$ M. G. Vincter, ${ }^{34}$ V. B. Vinogradov ${ }^{79}$ A. Vishwakarma, ${ }^{46}$ C. Vittori, ${ }^{23 b, 23 a}$ I. Vivarelli, ${ }^{156}$ M. Vogel, ${ }^{182}$ P. Vokac, ${ }^{142}$ S. E. von Buddenbrock, ${ }^{33 c}$ E. Von Toerne, ${ }^{24}$ V. Vorobel, ${ }^{143}$ K. Vorobev, ${ }^{112}$ M. Vos, ${ }^{174}$ J. H. Vossebeld, ${ }^{90}$ M. Vozak, ${ }^{100}$ N. Vranjes, ${ }^{16}$ M. Vranjes Milosavljevic, ${ }^{16}$ V. Vrba ${ }^{142}$ M. Vreeswijk, ${ }^{120}$ T. Šfiligoj, ${ }^{91}$ R. Vuillermet,${ }^{36}$ I. Vukotic ${ }^{37}$ T. Ženišs ${ }^{28 a}$ L. Živković, ${ }^{16}$ P. Wagner, ${ }^{24}$ W. Wagner, ${ }^{182}$ J. Wagner-Kuhr, ${ }^{114}$ H. Wahlberg, ${ }^{88}$ K. Wakamiya ${ }^{82}$ V. M. Walbrecht, ${ }^{115}$ J. Walder, ${ }^{89}$ R. Walker, ${ }^{114}$ S. D. Walker, ${ }^{93}$ W. Walkowiak, ${ }^{151}$ V. Wallangen, ${ }^{45 a, 45 b}$ A. M. Wang, ${ }^{59}$ C. Wang ${ }^{60 \mathrm{~b}}$ F. Wang, ${ }^{181}$ H. Wang, ${ }^{18}$ H. Wang, ${ }^{3}$ J. Wang, ${ }^{157}$ J. Wang, ${ }^{61 \mathrm{~b}}$ P. Wang, ${ }^{42}$ Q. Wang, ${ }^{128}$ R.-J. Wang, ${ }^{99}$ R. Wang, ${ }^{60 a}$ R. Wang, ${ }^{6}$ S. M. Wang, ${ }^{158}$ W. T. Wang, ${ }^{60 a}$ W. Wang, ${ }^{15 c, y y}$ W. X. Wang, ${ }^{60 a, y y}$ Y. Wang, ${ }^{60 a, z z}$ Z. Wang, ${ }^{60 c}$ C. Wanotayaroj, ${ }^{46}$ A. Warburton, ${ }^{103}$ C. P. Ward, ${ }^{32}$ D. R. Wardrope, ${ }^{94}$ N. Warrack, ${ }^{57}$ A. Washbrook, ${ }^{50}$ A. T. Watson, ${ }^{21}$ M. F. Watson, ${ }^{21}$ G. Watts, ${ }^{148}$ B. M. Waugh, ${ }^{94}$ A. F. Webb, ${ }^{11}$ S. Webb,${ }^{99}$ C. Weber, ${ }^{183}$ M. S. Weber,${ }^{20}$ S. A. Weber, ${ }^{34}$ S. M. Weber, ${ }^{61 a}$ A. R. Weidberg, ${ }^{135}$ J. Weingarten, ${ }^{47}$ M. Weirich, ${ }^{99}$ C. Weiser, ${ }^{52}$ P. S. Wells, ${ }^{36}$ T. Wenaus, ${ }^{29}$ T. Wengler, ${ }^{36}$ S. Wenig, ${ }^{36}$ N. Wermes, ${ }^{24}$ M. D. Werner, ${ }^{78}$ M. Wessels, ${ }^{61 \mathrm{a}}$ T. D. Weston, ${ }^{20}$ K. Whalen, ${ }^{131}$ N. L. Whallon, ${ }^{148}$ A. M. Wharton, ${ }^{89}$ A. S. White, ${ }^{105}$ A. White,${ }^{8}$ M. J. White, ${ }^{1}$ D. Whiteson, ${ }^{171}$ B. W. Whitmore,${ }^{89}$ F. J. Wickens, ${ }^{144}$ W. Wiedenmann, ${ }^{181}$ M. Wielers, ${ }^{144}$ N. Wieseotte, ${ }^{99}$ C. Wiglesworth, ${ }^{40}$ L. A. M. Wiik-Fuchs,${ }^{52}$ F. Wilk, ${ }^{100}$ H. G. Wilkens,${ }^{36}$ L. J. Wilkins, ${ }^{93}$ H. H. Williams, ${ }^{137}$ S. Williams, ${ }^{32}$ C. Willis, ${ }^{106}$ S. Willocq, ${ }^{102}$ J. A. Wilson, ${ }^{21}$ I. Wingerter-Seez, ${ }^{5}$ E. Winkels,${ }^{156}$ F. Winklmeier, ${ }^{131}$ O. J. Winston, ${ }^{156}$ B. T. Winter, ${ }^{52}$ M. Wittgen, ${ }^{153}$ M. Wobisch,${ }^{95}$ A. Wolf, ${ }^{99}$ T. M. H. Wolf, ${ }^{120}$ R. Wolff, ${ }^{101}$ R. W. Wölker, ${ }^{135}$ J. Wollrath, ${ }^{52}$ M. W. Wolter ${ }^{84}$ H. Wolters, ${ }^{140 a, 140 c}$ V. W. S. Wong, ${ }^{175}$ N. L. Woods, ${ }^{146}$ S. D. Worm, ${ }^{21}$ 
B. K. Wosiek, ${ }^{84}$ K. W. Woźniak ${ }^{84}$ K. Wraight,${ }^{57}$ S. L. Wu, ${ }^{181}$ X. Wu, ${ }^{54}$ Y. Wu ${ }^{60 a}$ T. R. Wyatt, ${ }^{100}$ B. M. Wynne,${ }^{50}$ S. Xella,${ }^{40}$ Z. Xi,${ }^{105}$ L. Xia, ${ }^{178}$ D. Xu ${ }^{15 a}$ H. Xu,${ }^{60 a, u u}$ L. Xu ${ }^{29}$ T. Xu, ${ }^{145}$ W. Xu,${ }^{105}$ Z. Xu,${ }^{60 b}$ Z. Xu, ${ }^{153}$ B. Yabsley, ${ }^{157}$ S. Yacoob,${ }^{33 a}$ K. Yajima, ${ }^{133}$ D. P. Yallup, ${ }^{94}$ D. Yamaguchi, ${ }^{165}$ Y. Yamaguchi, ${ }^{165}$ A. Yamamoto, ${ }^{81}$ T. Yamanaka, ${ }^{163}$ F. Yamane, ${ }^{82}$ M. Yamatani, ${ }^{163}$ T. Yamazaki, ${ }^{163}$ Y. Yamazaki, ${ }^{82}$ Z. Yan, ${ }^{25}$ H. J. Yang,${ }^{60,60 d}$ H. T. Yang, ${ }^{18}$ S. Yang, ${ }^{77}$ X. Yang, ${ }^{600,58}$ Y. Yang, ${ }^{163}$ W-M. Yao, ${ }^{18}$ Y. C. Yap, ${ }^{46}$ Y. Yasu, ${ }^{81}$ E. Yatsenko, ${ }^{60,60 d}$ J. Ye, ${ }^{42}$ S. Ye, ${ }^{29}$ I. Yeletskikh,${ }^{79}$ M. R. Yexley, ${ }^{89}$ E. Yigitbasi, ${ }^{25}$ K. Yorita, ${ }^{179}$ K. Yoshihara,${ }^{137}$ C. J. S. Young, ${ }^{36}$ C. Young, ${ }^{153}$ J. Yu, ${ }^{78}$ R. Yuan, ${ }^{60 b}$ X. Yue,${ }^{61 a}$ S. P. Y. Yuen, ${ }^{24}$ B. Zabinski, ${ }^{84}$ G. Zacharis, ${ }^{10}$ E. Zaffaroni, ${ }^{54}$ J. Zahreddine, ${ }^{136}$ A. M. Zaitsev, ${ }^{123,11}$ T. Zakareishvili, ${ }^{159 b}$ N. Zakharchuk, ${ }^{34}$ S. Zambito, ${ }^{59}$ D. Zanzi, ${ }^{36}$ D. R. Zaripovas, ${ }^{57}$ S. V. Zeißner, ${ }^{47}$ C. Zeitnitz, ${ }^{182}$ G. Zemaityte, ${ }^{135}$ J. C. Zeng, ${ }^{173}$ O. Zenin, ${ }^{123}$ D. Zerwas, ${ }^{132}$ M. Zgubič ${ }^{135}$ D. F. Zhang, ${ }^{15 b}$ F. Zhang, ${ }^{181}$ G. Zhang, ${ }^{60 a}$ G. Zhang, ${ }^{15 b}$ H. Zhang, ${ }^{15 c}$ J. Zhang, ${ }^{6}$ L. Zhang, ${ }^{15 c}$ L. Zhang, ${ }^{60 a}$ M. Zhang, ${ }^{173}$ R. Zhang, ${ }^{60 a}$ R. Zhang, ${ }^{24}$ X. Zhang, ${ }^{60 b}$ Y. Zhang, ${ }^{15 a, 15 d}$ Z. Zhang, ${ }^{63 a}$ Z. Zhang, ${ }^{132}$ P. Zhao, ${ }^{49}$ Y. Zhao, ${ }^{60 b}$ Z. Zhao, ${ }^{60 a}$ A. Zhemchugov, ${ }^{79}$ Z. Zheng, ${ }^{105}$ D. Zhong, ${ }^{173}$ B. Zhou, ${ }^{105}$ C. Zhou, ${ }^{181}$ M. S. Zhou, ${ }^{15 a, 15 d}$ M. Zhou, ${ }^{155}$ N. Zhou, ${ }^{60 \mathrm{c}}$ Y. Zhou, ${ }^{7}$ C. G. Zhu, ${ }^{60 \mathrm{~b}}$ H. L. Zhu, ${ }^{60 \mathrm{a}}$ H. Zhu, ${ }^{15 \mathrm{a}}$ J. Zhu, ${ }^{105}$ Y. Zhu, ${ }^{60 \mathrm{a}}$ X. Zhuang, ${ }^{15 \mathrm{a}}$ K. Zhukov, ${ }^{110}$ V. Zhulanov, ${ }^{122 b, 122 a}$ D. Zieminska, ${ }^{65}$ N. I. Zimine, ${ }^{79}$ S. Zimmermann, ${ }^{52}$ Z. Zinonos, ${ }^{115}$ M. Ziolkowski, ${ }^{151}$ G. Zobernig, ${ }^{181}$ A. Zoccoli, ${ }^{23 b, 23 a}$ K. Zoch, ${ }^{53}$ T. G. Zorbas, ${ }^{149}$ R. Zou, ${ }^{37}$ and L. Zwalinski ${ }^{36}$

(ATLAS Collaboration)

\footnotetext{
${ }^{1}$ Department of Physics, University of Adelaide, Adelaide, Australia

${ }^{2}$ Physics Department, SUNY Albany, Albany, New York, USA

${ }^{3}$ Department of Physics, University of Alberta, Edmonton, Alberta, Canada

${ }^{4 a}$ Department of Physics, Ankara University, Ankara, Turkey

${ }^{4 \mathrm{~b}}$ Istanbul Aydin University, Istanbul, Turkey

${ }^{4 \mathrm{c}}$ Division of Physics, TOBB University of Economics and Technology, Ankara, Turkey

${ }^{5}$ LAPP, Université Grenoble Alpes, Université Savoie Mont Blanc, CNRS/IN2P3, Annecy, France

${ }^{6}$ High Energy Physics Division, Argonne National Laboratory, Argonne, Illinois, USA

${ }^{7}$ Department of Physics, University of Arizona, Tucson, Arizona, USA

${ }^{8}$ Department of Physics, University of Texas at Arlington, Arlington, Texas, USA

${ }^{9}$ Physics Department, National and Kapodistrian University of Athens, Athens, Greece

${ }^{10}$ Physics Department, National Technical University of Athens, Zografou, Greece

${ }^{11}$ Department of Physics, University of Texas at Austin, Austin, Texas, USA

${ }^{12 a}$ Bahcesehir University, Faculty of Engineering and Natural Sciences, Istanbul, Turkey

${ }^{12 \mathrm{~b}}$ Istanbul Bilgi University, Faculty of Engineering and Natural Sciences, Istanbul, Turkey

${ }^{12 \mathrm{c}}$ Department of Physics, Bogazici University, Istanbul, Turkey

${ }^{12 \mathrm{~d}}$ Department of Physics Engineering, Gaziantep University, Gaziantep, Turkey

${ }^{13}$ Institute of Physics, Azerbaijan Academy of Sciences, Baku, Azerbaijan

${ }^{14}$ Institut de Física d'Altes Energies (IFAE), Barcelona Institute of Science and Technology, Barcelona, Spain

${ }^{15 a}$ Institute of High Energy Physics, Chinese Academy of Sciences, Beijing, China

${ }^{15 \mathrm{~b}}$ Physics Department, Tsinghua University, Beijing, China

${ }^{15 \mathrm{c}}$ Department of Physics, Nanjing University, Nanjing, China

${ }^{15 \mathrm{~d}}$ University of Chinese Academy of Science (UCAS), Beijing, China

${ }^{16}$ Institute of Physics, University of Belgrade, Belgrade, Serbia

${ }^{17}$ Department for Physics and Technology, University of Bergen, Bergen, Norway

${ }^{18}$ Physics Division, Lawrence Berkeley National Laboratory and University of California, Berkeley, California, USA

${ }^{19}$ Institut für Physik, Humboldt Universität zu Berlin, Berlin, Germany

${ }^{20}$ Albert Einstein Center for Fundamental Physics and Laboratory for High Energy Physics, University of Bern, Bern, Switzerland

${ }^{21}$ School of Physics and Astronomy, University of Birmingham, Birmingham, United Kingdom

${ }^{22}$ Facultad de Ciencias y Centro de Investigaciónes, Universidad Antonio Nariño, Bogota, Colombia

${ }^{23 a}$ INFN Bologna and Universita' di Bologna, Dipartimento di Fisica, Italy

${ }^{23 \mathrm{~b}}$ INFN Sezione di Bologna, Italy

${ }^{24}$ Physikalisches Institut, Universität Bonn, Bonn, Germany

${ }^{25}$ Department of Physics, Boston University, Boston, Massachusetts, USA

${ }^{26}$ Department of Physics, Brandeis University, Waltham, Massachusetts, USA

${ }^{27}$ Transilvania University of Brasov, Brasov, Romania

${ }^{27 \mathrm{~b}}$ Horia Hulubei National Institute of Physics and Nuclear Engineering, Bucharest, Romania

${ }^{27 c}$ Department of Physics, Alexandru Ioan Cuza University of Iasi, Iasi, Romania
} 
${ }^{27 \mathrm{~d}}$ National Institute for Research and Development of Isotopic and Molecular Technologies, Physics Department, Cluj-Napoca, Romania

${ }^{27 \mathrm{e}}$ University Politehnica Bucharest, Bucharest, Romania

${ }^{27 \mathrm{f}}$ West University in Timisoara, Timisoara, Romania

${ }^{28 \mathrm{a}}$ Faculty of Mathematics, Physics and Informatics, Comenius University, Bratislava, Slovak Republic

${ }^{28 \mathrm{~b}}$ Department of Subnuclear Physics, Institute of Experimental Physics of the Slovak Academy of Sciences, Kosice, Slovak Republic

${ }^{29}$ Physics Department, Brookhaven National Laboratory, Upton, New York, USA

${ }^{30}$ Departamento de Física, Universidad de Buenos Aires, Buenos Aires, Argentina

${ }^{31}$ California State University, California, USA

${ }^{32}$ Cavendish Laboratory, University of Cambridge, Cambridge, United Kingdom

${ }^{33 a}$ Department of Physics, University of Cape Town, Cape Town, South Africa

${ }^{33 \mathrm{~b}}$ Department of Mechanical Engineering Science, University of Johannesburg, Johannesburg, South Africa

${ }^{33 \mathrm{c}}$ School of Physics, University of the Witwatersrand, Johannesburg, South Africa

${ }^{34}$ Department of Physics, Carleton University, Ottawa, Ontario, Canada

${ }^{35 a}$ Faculté des Sciences Ain Chock, Réseau Universitaire de Physique des Hautes Energies-Université Hassan II, Casablanca, Morocco

${ }^{35 \mathrm{~b}}$ Faculté des Sciences, Université Ibn-Tofail, Kénitra, Morocco

${ }^{35 c}$ Faculté des Sciences Semlalia, Université Cadi Ayyad, LPHEA-Marrakech, Morocco

${ }^{35 \mathrm{~d}}$ Faculté des Sciences, Université Mohamed Premier and LPTPM, Oujda, Morocco

${ }^{35}$ Faculté des sciences, Université Mohammed V, Rabat, Morocco

${ }^{36}$ CERN, Geneva, Switzerland

${ }^{37}$ Enrico Fermi Institute, University of Chicago, Chicago, Illinois, USA

${ }^{38}$ LPC, Université Clermont Auvergne, CNRS/IN2P3, Clermont-Ferrand, France

${ }^{39}$ Nevis Laboratory, Columbia University, Irvington, New York, USA

${ }^{40}$ Niels Bohr Institute, University of Copenhagen, Copenhagen, Denmark

${ }^{41 a}$ Dipartimento di Fisica, Università della Calabria, Rende, Italy

${ }^{41 \mathrm{~b}}$ INFN Gruppo Collegato di Cosenza, Laboratori Nazionali di Frascati, Italy

${ }^{42}$ Physics Department, Southern Methodist University, Dallas, Texas, USA

${ }^{43}$ Physics Department, University of Texas at Dallas, Richardson, Texas, USA

${ }^{44}$ National Centre for Scientific Research "Demokritos", Agia Paraskevi, Greece

${ }^{45 a}$ Department of Physics, Stockholm University, Sweden

${ }^{45 \mathrm{~b}}$ Oskar Klein Centre, Stockholm, Sweden

${ }^{46}$ Deutsches Elektronen-Synchrotron DESY, Hamburg and Zeuthen, Germany

${ }^{47}$ Lehrstuhl für Experimentelle Physik IV, Technische Universität Dortmund, Dortmund, Germany

${ }^{48}$ Institut für Kern- und Teilchenphysik, Technische Universität Dresden, Dresden, Germany

${ }^{49}$ Department of Physics, Duke University, Durham, North Carolina, USA

${ }^{50}$ SUPA-School of Physics and Astronomy, University of Edinburgh, Edinburgh, United Kingdom

${ }^{51}$ INFN e Laboratori Nazionali di Frascati, Frascati, Italy

${ }^{52}$ Physikalisches Institut, Albert-Ludwigs-Universität Freiburg, Freiburg, Germany

${ }^{53}$ II. Physikalisches Institut, Georg-August-Universität Göttingen, Göttingen, Germany

${ }^{54}$ Département de Physique Nucléaire et Corpusculaire, Université de Genève, Genève, Switzerland

${ }^{55}$ Dipartimento di Fisica, Università di Genova, Genova, Italy

${ }^{55 \mathrm{~b}}$ INFN Sezione di Genova, Italy

${ }^{56}$ II. Physikalisches Institut, Justus-Liebig-Universität Giessen, Giessen, Germany

${ }^{57}$ SUPA-School of Physics and Astronomy, University of Glasgow, Glasgow, United Kingdom

${ }^{58}$ LPSC, Université Grenoble Alpes, CNRS/IN2P3, Grenoble INP, Grenoble, France

${ }^{59}$ Laboratory for Particle Physics and Cosmology, Harvard University, Cambridge, Massachusetts, USA

${ }^{60 \mathrm{a}}$ Department of Modern Physics and State Key Laboratory of Particle Detection and Electronics,

University of Science and Technology of China, Hefei, China

${ }^{60 \mathrm{~b}}$ Institute of Frontier and Interdisciplinary Science and Key Laboratory of Particle Physics and Particle Irradiation (MOE), Shandong University, Qingdao, China

${ }^{60 \mathrm{c} S c h o o l}$ of Physics and Astronomy, Shanghai Jiao Tong University, KLPPAC-MoE, SKLPPC, Shanghai, China

${ }^{60 \mathrm{~d}}$ Tsung-Dao Lee Institute, Shanghai, China

${ }^{61 a}$ Kirchhoff-Institut für Physik, Ruprecht-Karls-Universität Heidelberg, Heidelberg, Germany

${ }^{61 \mathrm{~b}}$ Physikalisches Institut, Ruprecht-Karls-Universität Heidelberg, Heidelberg, Germany

${ }^{62}$ Faculty of Applied Information Science, Hiroshima Institute of Technology, Hiroshima, Japan

${ }^{63 a}$ Department of Physics, Chinese University of Hong Kong, Shatin, N.T., Hong Kong, China

${ }^{63 \mathrm{~b}}$ Department of Physics, University of Hong Kong, Hong Kong, China

${ }^{63 \mathrm{c}}$ Department of Physics and Institute for Advanced Study, Hong Kong University of Science and Technology, Clear Water Bay, Kowloon, Hong Kong, China 
${ }^{108}$ Research Institute for Nuclear Problems of Byelorussian State University, Minsk, Belarus

${ }^{109}$ Group of Particle Physics, University of Montreal, Montreal, Quebec, Canada

${ }^{110}$ P.N. Lebedev Physical Institute of the Russian Academy of Sciences, Moscow, Russia

${ }^{111}$ Institute for Theoretical and Experimental Physics of the National Research Centre Kurchatov Institute, Moscow, Russia

${ }^{112}$ National Research Nuclear University MEPhI, Moscow, Russia

${ }^{113}$ D.V. Skobeltsyn Institute of Nuclear Physics, M.V. Lomonosov Moscow State University, Moscow, Russia

${ }^{114}$ Fakultät für Physik, Ludwig-Maximilians-Universität München, München, Germany

${ }^{115}$ Max-Planck-Institut für Physik (Werner-Heisenberg-Institut), München, Germany

${ }^{116}$ Nagasaki Institute of Applied Science, Nagasaki, Japan

${ }^{117}$ Graduate School of Science and Kobayashi-Maskawa Institute, Nagoya University, Nagoya, Japan

${ }^{118}$ Department of Physics and Astronomy, University of New Mexico, Albuquerque, New Mexico, USA

${ }^{119}$ Institute for Mathematics, Astrophysics and Particle Physics, Radboud University Nijmegen/Nikhef, Nijmegen, Netherlands

${ }^{120}$ Nikhef National Institute for Subatomic Physics and University of Amsterdam, Amsterdam, Netherlands

${ }^{121}$ Department of Physics, Northern Illinois University, DeKalb, Illinois, USA

${ }^{122 a}$ Budker Institute of Nuclear Physics and NSU, SB RAS, Novosibirsk, Russia

${ }^{122 \mathrm{~b}}$ Novosibirsk State University Novosibirsk, Russia

${ }^{123}$ Institute for High Energy Physics of the National Research Centre Kurchatov Institute, Protvino, Russia

${ }^{124}$ Department of Physics, New York University, New York, New York, USA

${ }^{125}$ Ochanomizu University, Otsuka, Bunkyo-ku, Tokyo, Japan

${ }^{126}$ The Ohio State University, Columbus, Ohio, USA

${ }^{127}$ Faculty of Science, Okayama University, Okayama, Japan

${ }^{128}$ Homer L. Dodge Department of Physics and Astronomy, University of Oklahoma, Norman, Oklahoma, USA

${ }^{129}$ Department of Physics, Oklahoma State University, Stillwater, Oklahoma, USA

${ }^{130}$ Palacký University, RCPTM, Joint Laboratory of Optics, Olomouc, Czech Republic

${ }^{131}$ Center for High Energy Physics, University of Oregon, Eugene, Oregon, USA

${ }^{132}$ LAL, Université Paris-Sud, CNRS/IN2P3, Université Paris-Saclay, Orsay, France

${ }^{133}$ Graduate School of Science, Osaka University, Osaka, Japan

${ }^{134}$ Department of Physics, University of Oslo, Oslo, Norway

${ }^{135}$ Department of Physics, Oxford University, Oxford, United Kingdom

${ }^{136}$ LPNHE, Sorbonne Université, Paris Diderot Sorbonne Paris Cité, CNRS/IN2P3, Paris, France

${ }^{137}$ Department of Physics, University of Pennsylvania, Philadelphia, Pennsylvania, USA

${ }^{138}$ Konstantinov Nuclear Physics Institute of National Research Centre "Kurchatov Institute”, PNPI, St. Petersburg, Russia

${ }^{139}$ Department of Physics and Astronomy, University of Pittsburgh, Pittsburgh, Pennsylvania, USA

${ }^{140 a}$ Laboratório de Instrumentação e Física Experimental de Partículas-LIP, Portugal

${ }^{140 \mathrm{~b}}$ Departamento de Física, Faculdade de Ciências, Universidade de Lisboa, Lisboa, Portugal

${ }^{140 \mathrm{c}}$ Departamento de Física, Universidade de Coimbra, Coimbra, Portugal

${ }^{140 \mathrm{~d}}$ Centro de Física Nuclear da Universidade de Lisboa, Lisboa, Portugal

${ }^{140 \mathrm{e}}$ Departamento de Física, Universidade do Minho, Braga, Portugal

${ }^{140 \mathrm{f}}$ Universidad de Granada, Granada, Spain

${ }^{140 \mathrm{~g} D e p}$ Física and CEFITEC of Faculdade de Ciências e Tecnologia, Universidade Nova de Lisboa, Caparica, Portugal

${ }^{141}$ Institute of Physics of the Czech Academy of Sciences, Prague, Czech Republic

${ }^{142}$ Czech Technical University in Prague, Prague, Czech Republic

${ }^{143}$ Charles University, Faculty of Mathematics and Physics, Prague, Czech Republic

${ }^{144}$ Particle Physics Department, Rutherford Appleton Laboratory, Didcot, United Kingdom

${ }^{145}$ IRFU, CEA, Université Paris-Saclay, Gif-sur-Yvette, France

${ }^{146}$ Santa Cruz Institute for Particle Physics, University of California Santa Cruz, Santa Cruz, California, USA

${ }^{147 a}$ Departamento de Física, Pontificia Universidad Católica de Chile, Santiago, Chile

${ }^{147 b}$ Departamento de Física, Universidad Técnica Federico Santa María, Valparaíso, Chile

${ }^{148}$ Department of Physics, University of Washington, Seattle, Washington, USA

${ }^{149}$ Department of Physics and Astronomy, University of Sheffield, Sheffield, United Kingdom

${ }^{150}$ Department of Physics, Shinshu University, Nagano, Japan

${ }^{151}$ Department Physik, Universität Siegen, Siegen, Germany

${ }^{152}$ Department of Physics, Simon Fraser University, Burnaby, British Columbia, Canada

${ }^{153}$ SLAC National Accelerator Laboratory, Stanford, California, USA

${ }^{154}$ Physics Department, Royal Institute of Technology, Stockholm, Sweden

${ }^{155}$ Departments of Physics and Astronomy, Stony Brook University, Stony Brook, New York, USA

${ }^{156}$ Department of Physics and Astronomy, University of Sussex, Brighton, United Kingdom

${ }^{157}$ School of Physics, University of Sydney, Sydney, Australia

${ }^{158}$ Institute of Physics, Academia Sinica, Taipei, Taiwan

${ }^{159 a}$ E. Andronikashvili Institute of Physics, Iv. Javakhishvili Tbilisi State University, Tbilisi, Georgia 


\footnotetext{
${ }^{159 \mathrm{~b}}$ High Energy Physics Institute, Tbilisi State University, Tbilisi, Georgia

${ }^{160}$ Department of Physics, Technion, Israel Institute of Technology, Haifa, Israel

${ }^{161}$ Raymond and Beverly Sackler School of Physics and Astronomy, Tel Aviv University, Tel Aviv, Israel

${ }^{162}$ Department of Physics, Aristotle University of Thessaloniki, Thessaloniki, Greece

${ }^{163}$ International Center for Elementary Particle Physics and Department of Physics, University of Tokyo, Tokyo, Japan

${ }^{164}$ Graduate School of Science and Technology, Tokyo Metropolitan University, Tokyo, Japan

${ }^{165}$ Department of Physics, Tokyo Institute of Technology, Tokyo, Japan

${ }^{166}$ Tomsk State University, Tomsk, Russia

${ }^{167}$ Department of Physics, University of Toronto, Toronto, Ontario, Canada

${ }^{168 a}$ TRIUMF, Vancouver, British Columbia, Canada

${ }^{168 \mathrm{~b}}$ Department of Physics and Astronomy, York University, Toronto, Ontario, Canada

${ }^{169}$ Division of Physics and Tomonaga Center for the History of the Universe, Faculty of Pure and Applied Sciences, University of Tsukuba, Tsukuba, Japan

${ }^{170}$ Department of Physics and Astronomy, Tufts University, Medford, Massachusetts, USA

${ }^{171}$ Department of Physics and Astronomy, University of California Irvine, Irvine, California, USA

${ }^{172}$ Department of Physics and Astronomy, University of Uppsala, Uppsala, Sweden

${ }^{173}$ Department of Physics, University of Illinois, Urbana, Illinois, USA

${ }^{174}$ Instituto de Física Corpuscular (IFIC), Centro Mixto Universidad de Valencia-CSIC, Valencia, Spain

${ }^{175}$ Department of Physics, University of British Columbia, Vancouver, British Columbia, Canada

${ }^{177}$ Department of Physics and Astronomy, University of Victoria, Victoria, British Columbia, Canada

${ }^{177}$ Fakultät für Physik und Astronomie, Julius-Maximilians-Universität Würzburg, Würzburg, Germany

${ }^{178}$ Department of Physics, University of Warwick, Coventry, United Kingdom

${ }^{179}$ Waseda University, Tokyo, Japan

${ }^{180}$ Department of Particle Physics, Weizmann Institute of Science, Rehovot, Israel

${ }^{181}$ Department of Physics, University of Wisconsin, Madison, Wisconsin, USA

${ }^{182}$ Fakultät für Mathematik und Naturwissenschaften, Fachgruppe Physik, Bergische Universität Wuppertal, Wuppertal, Germany

${ }^{183}$ Department of Physics, Yale University, New Haven, Connecticut, USA

${ }^{184}$ Yerevan Physics Institute, Yerevan, Armenia

${ }^{\mathrm{a}}$ Deceased.

${ }^{\mathrm{b}}$ Also at Department of Physics, King's College London, London, United Kingdom.

${ }^{c}$ Also at Istanbul University, Department of Physics, Istanbul, Turkey.

${ }^{\mathrm{d}}$ Also at Instituto de Fisica Teorica, IFT-UAM/CSIC, Madrid, Spain.

${ }^{\mathrm{e}}$ Also at TRIUMF, Vancouver, British Columbia, Canada.

${ }^{\mathrm{f}}$ Also at Department of Physics and Astronomy, University of Louisville, Louisville, Kentucky, USA.

${ }^{\mathrm{g}}$ Also at Physics Department, An-Najah National University, Nablus, Palestine.

${ }^{\mathrm{h}}$ Also at Department of Physics, California State University, Fresno, USA.

${ }^{\mathrm{i}}$ Also at Department of Physics, University of Fribourg, Fribourg, Switzerland.

${ }^{j}$ Also at Physics Dept, University of South Africa, Pretoria, South Africa.

${ }^{\mathrm{k}}$ Also at Departament de Fisica de la Universitat Autonoma de Barcelona, Barcelona, Spain.

${ }^{1}$ Also at Tomsk State University, Tomsk, and Moscow Institute of Physics and Technology State University, Dolgoprudny, Russia.

${ }^{\mathrm{m}}$ Also at The Collaborative Innovation Center of Quantum Matter (CICQM), Beijing, China.

${ }^{\mathrm{n}}$ Also at Departamento de Física, Instituto Superior Técnico, Universidade de Lisboa, Lisboa, Portugal.

${ }^{\circ}$ Also at Universita di Napoli Parthenope, Napoli, Italy.

${ }^{\mathrm{p}}$ Also at Institute of Particle Physics (IPP), Canada.

${ }^{\mathrm{q}}$ Also at Department of Physics, University of Adelaide, Adelaide, Australia.

${ }^{r}$ Also at Department of Physics, St. Petersburg State Polytechnical University, St. Petersburg, Russia.

${ }^{\mathrm{s}}$ Also at Department of Financial and Management Engineering, University of the Aegean, Chios, Greece.

${ }^{t}$ Also at Centre for High Performance Computing, CSIR Campus, Rosebank, Cape Town, South Africa.

"Also at Department of Physics, California State University, East Bay, USA.

${ }^{v}$ Also at Institucio Catalana de Recerca i Estudis Avancats, ICREA, Barcelona, Spain.

${ }^{w}$ Also at Department of Physics, University of Michigan, Ann Arbor, Michigan, USA.

${ }^{x}$ Also at LAL, Université Paris-Sud, CNRS/IN2P3, Université Paris-Saclay, Orsay, France.

${ }^{\mathrm{y}}$ Also at Graduate School of Science, Osaka University, Osaka, Japan.

${ }^{\mathrm{z}}$ Also at Physikalisches Institut, Albert-Ludwigs-Universität Freiburg, Freiburg, Germany.

${ }^{\text {aa } A l s o ~ a t ~ I n s t i t u t e ~ o f ~ P h y s i c s, ~ A z e r b a i j a n ~ A c a d e m y ~ o f ~ S c i e n c e s, ~ B a k u, ~ A z e r b a i j a n . ~}$

${ }^{\mathrm{bb}}$ Also at Institute for Mathematics, Astrophysics and Particle Physics, Radboud University Nijmegen/Nikhef, Nijmegen, Netherlands.

${ }^{c c}$ Also at Institute of Theoretical Physics, Ilia State University, Tbilisi, Georgia.

${ }^{\mathrm{dd}}$ Also at CERN, Geneva, Switzerland.
} 


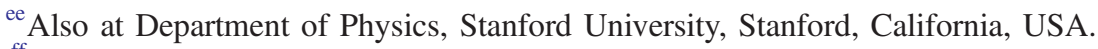

${ }^{\mathrm{ff}}$ Also at Manhattan College, New York, New York, USA.

${ }^{g g}$ Also at Joint Institute for Nuclear Research, Dubna, Russia.

hh Also at Hellenic Open University, Patras, Greece.

${ }^{\text {ii } A l s o ~ a t ~ T h e ~ C i t y ~ C o l l e g e ~ o f ~ N e w ~ Y o r k, ~ N e w ~ Y o r k, ~ N e w ~ Y o r k, ~ U S A . ~}$

${ }^{\mathrm{jj}}$ Also at Institute of High Energy Physics, Chinese Academy of Sciences, Beijing, China.

${ }^{\mathrm{kk}}$ Also at Department of Physics, California State University, Sacramento, USA.

${ }^{1 l}$ Also at Moscow Institute of Physics and Technology State University, Dolgoprudny, Russia.

${ }^{\mathrm{mm}}$ Also at Département de Physique Nucléaire et Corpusculaire, Université de Genève, Genève, Switzerland.

${ }^{\mathrm{nn}}$ Also at Department of Physics and Astronomy, University of Sheffield, Sheffield, United Kingdom.

${ }^{\circ o}$ Also at Louisiana Tech University, Ruston, Louisiana, USA.

${ }^{\mathrm{pp}}$ Also at School of Physics, Sun Yat-sen University, Guangzhou, China.

${ }^{\mathrm{qq}}$ Also at Institute for Nuclear Research and Nuclear Energy (INRNE) of the Bulgarian Academy of Sciences, Sofia, Bulgaria.

${ }^{\mathrm{rr}}$ Also at Faculty of Physics, M.V. Lomonosov Moscow State University, Moscow, Russia.

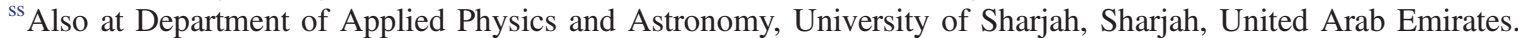

${ }^{\text {tt}}$ Also at Institut für Experimentalphysik, Universität Hamburg, Hamburg, Germany.

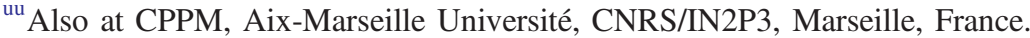

${ }^{v v}$ Also at National Research Nuclear University MEPhI, Moscow, Russia.

${ }^{w w}$ Also at Institute for Particle and Nuclear Physics, Wigner Research Centre for Physics, Budapest, Hungary.

${ }^{\mathrm{xx}}$ Also at Giresun University, Faculty of Engineering, Giresun, Turkey.

${ }^{\text {yy }}$ Also at Institute of Physics, Academia Sinica, Taipei, Taiwan.

${ }^{\mathrm{zz}}$ Also at LPNHE, Sorbonne Université, Paris Diderot Sorbonne Paris Cité, CNRS/IN2P3, Paris, France
} 